\title{
Nonmyopic Optimal Portfolios in Viable Markets *
}

\author{
Jakša Cvitanić ${ }^{\dagger}$ and Semyon Malamud ${ }^{\ddagger}$
}

October 6, 2010

\begin{abstract}
We provide a representation for the nonmyopic optimal portfolio of an agent consuming only at the terminal horizon when the single state variable follows a general diffusion process and the market consists of one risky asset and a risk-free asset. The key term of our representation is a new object that we call the "rate of macroeconomic fluctuation" whose properties are fundamental for the portfolio dynamics. We show that, under natural cyclicality conditions, (i) the agent's hedging demand is positive (negative) when the product of his prudence and risk tolerance is below (above) 2 and (ii) the portfolio weights decrease in risk aversion. We apply our results to study a general continuous-time capital asset pricing model and show that under the same cyclicality conditions, the market price of risk is countercyclical and the price of the risky asset exhibits excess volatility.
\end{abstract}

Keywords: heterogeneous agents, nonmyopic optimal portfolios, hedging demand, equilibrium

JEL Classification: D53, G11, G12

*The research of J. Cvitanić was supported in part by NSF grant DMS 06-31298. The research of S. Malamud was supported in part by NCCR FINRISK, Project D1. We thank Patrick Bolton, Jerome Detemple, Bernard Dumas, Campbell Harvey, Julien Hugonnier, Elyes Jouini, Loriano Mancini, Rajnish Mehra, and especially Erwan Morellec for helpful comments, as well as audiences at a number of seminars and conferences. Existing errors are our sole responsibility. A previous version of this paper was titled "Equilibrium Asset Pricing and Portfolio Choice with Heterogeneous Preferences".

${ }^{\dagger}$ Caltech, Division of Humanities and Social Sciences, M/C 228-77, 1200 E. California Blvd. Pasadena, CA 91125. Ph: (626) 395-1784. E-mail: cvitanic@hss.caltech.edu

${ }^{\ddagger}$ Swiss Finance Institute, EPF Lausanne. E-mail: semyon.malamud@epfl.ch 


\section{Introduction}

The problem of optimal portfolio allocation for a long-run investor, in a market with one risky asset and a risk-free asset, is of fundamental importance in financial economics. When the market price of risk (MPR) and/or interest rates are stochastic, the optimal portfolio is inherently nonmyopic and depends on not only current asset returns but also their future fluctuations. As Merton $(1969,1971)$ indicates, the optimal portfolio can be decomposed into two components: a myopic, instantaneously mean-variance efficient component and a strategic, nonmyopic component responsible for hedging against future changes in the investment opportunity set. Several recent empirical studies indicate that the hedging component may represent a huge fraction (40-100\%) of total portfolio holdings. ${ }^{1}$ However, since it is generally not possible to derive closed-form expressions for nonmyopic optimal portfolios, very little is known about their dependence on the agent's risk preferences and the dynamic properties of asset returns.

This paper provides representations for nonmyopic optimal portfolios of an agent consuming only at the terminal horizon when the single state variable follows a general diffusion process. The key additional assumption we make is that the state price density is a function of the underlying state variable. This assumption holds true if and only if the price of the risky asset in the economy and the interest rate of the risk-free asset are viable; that is, they can be realized in a complete market equilibrium within the capital asset pricing model (CAPM) if the representative agent is chosen appropriately. This makes our results perfectly suited to equilibrium analysis, which we present in the second part of the paper. We use the obtained representations to generate empirical predictions and establish various properties of the nonmyopic portfolio strategies.

We introduce a new object that we call the "rate of macroeconomic fluctuation" and show that the rate of macroeconomic fluctuation is the key element of all representations derived in this paper, both in the single-agent partial equilibrium setting and in the general equilibrium setting. The rate of macroeconomic fluctuation is an intrinsic characteristic of the state variable dynamics and has the following interesting property: in equilibrium, the risk-neutral drift of the MPR is independent of the form of the agents' utility functions and is always equal to the rate of macroeconomic fluctuation. As a result, the rate of macroeconomic fluctuation can be directly estimated from the data as the difference between the drift of the MPR and the product of the MPR and its

\footnotetext{
${ }^{1}$ See Campbell and Viceira (1999) and Barberis (2000).
} 
volatility. $^{2}$

We show that the intertemporal hedge against the MPR fluctuations has two components: a wealth hedge and a risk tolerance hedge. The wealth (risk tolerance) hedge is given by the covariance of the agent's future wealth (risk tolerance) with the future MPR, discounted by the rate of macroeconomic fluctuation.

Our representation allows us to determine the precise dependence of the optimal portfolios on the market's and the agent's characteristics. In particular, we show that the optimal investment in the risky asset is monotonically decreasing in risk aversion in the sense of Ross (1981) as long as the rate of macroeconomic fluctuation is constant. This result is quite striking in its generality, as it holds for arbitrary utilities, arbitrary dividend volatility and arbitrary stochastic interest rates. It also generates this empirical prediction: if the volatility of the rate of macroeconomic fluctuation is relatively low, more risk averse agents should invest less in the risky asset.

We show that, when the MPR is countercyclical, the hedging demand is positive if and only if the product of the agent's prudence and risk tolerance is below 2. We provide an intuitive explanation of this non-obvious result and relate it to the precautionary savings motive.

If we adopt the common hypothesis that agents have hyperbolic absolute risk aversion (HARA) utilities, our results generate the prediction that the variation in the MPR hedging demand of an agent is explained by the variation in the co-movement of agent's wealth and the discounted MPR. This prediction can be tested directly using Panel Study of Income Dynamics (PSID) data. It would allow us to gain a deeper understanding of the joint fluctuations of household wealth and optimal portfolios. For example, Brunnermeier and Nagel (2008) find that the fraction of wealth that households invest in risky assets does not change with the level of wealth. They use this finding to conclude that households' relative risk aversion is constant. However, if this risk aversion is different from 1, the optimal portfolio must be nonmyopic and contain a hedging component. Given our representation of hedging demand, the findings of Brunnermeier and Nagel (2008) imply, in our model, that the degree of co-movement of households' wealth with the MPR is stable and does not fluctuate over time. It would be of interest to test this prediction and determine the degree to which investment by households is driven by the nonmyopic demand.

\footnotetext{
${ }^{2}$ That is, the MPR $\lambda$ follows the dynamics $d \lambda_{t}=\lambda_{t}\left(\mu_{t}^{\lambda} d t+\sigma_{t}^{\lambda} d B_{t}\right)$ with the drift $\mu_{t}^{\lambda}=\rho_{t}+\lambda_{t} \sigma_{t}^{\lambda}$ where $\rho_{t}$ is the rate of macroeconomic fluctuation.
} 
In the section on equilibrium dynamics, we provide tight conditions for MPR countercyclicality and for the positivity of the nonmyopic (excess) volatility. We show that, when interest rate volatility is small, MPR is countercyclical if the product of the aggregate risk aversion and dividend volatility is countercyclical and the rate of macroeconomic fluctuation is procyclical. This result extends the characterization of He and Leland (1993) to general dividend processes and stochastic interest rates. In particular, we show, in contrast to the sufficient conditions in the framework of that paper, that when the state variable follows a general diffusion process even countercyclicality of both dividend volatility and aggregate risk aversion may not be sufficient for MPR to be countercyclical, as its equilibrium behavior depends on the dynamics of the rate of macroeconomic fluctuation.

In complete analogy with the optimal portfolios, we show that the volatility of the risky asset can be decomposed into myopic and nonmyopic components. The latter plays the role of "excess" volatility and is positive under the same conditions for which MPR is countercyclical. As above, these conditions are tight.

In the special case when the log-dividend follows an autoregressive process and all agents have constant relative risk aversion (CRRA) preferences, our results are strengthened further. We find explicit bounds on MPR, excess volatility, risk-return tradeoff and the size of equilibrium optimal portfolios in terms of the spread between the highest and the lowest risk aversion in the economy. This is a natural measure of heterogeneity, and our bounds can be used to estimate this measure from data.

We conclude the introduction with a discussion of the related literature. Our paper is most closely related to the fundamental work by Detemple, Garcia, and Rindisbacher (2003) and Detemple and Rindisbacher (2005, 2009), who were the first to use Malliavian calculus to derive general representations for intertemporal hedging demand with general utilities and a general multi-dimensional diffusion state variable. In our paper, the assumption that the market is viable leads to a particularly simple and intuitive representation of optimal portfolios and allows us to establish several important economic properties of the latter.

Gollier and Zeckhauser (2002), Aura, Diamond and Geanakoplos (2002) and Gollier (2004) investigate nonmyopic portfolios in a discrete time two-period model. In particular, Gollier (2004) studies the effect of first- and second-order predictability in returns in the sign of the hedging demand. Our introduction of the rate of macroeconomic fluctuation allows us to determine the sign of hedging demand for very general 
continuous-time dynamic settings with an arbitrary time horizon.

A substantial body of literature is devoted to the partial equilibrium continuoustime nonmyopic optimal portfolio behavior in complete and incomplete markets when utilities belong to the HARA class and stock prices follow an affine process. This specification allows for closed-form solutions for optimal portfolios (see Kim and Omberg 1996 and Wachter 2002).

Most existing work devoted to standard CAPM with heterogeneous risk preferences has been done in special models with two CRRA agents only. Dumas (1989) considers a production economy of that type. In this case, the risky asset returns coincide with the exogenously specified returns on production technology, and only the risk-free rate is determined endogenously. Wang (1996) studies the term structure of interest rates in an economy populated by two CRRA agents, maximizing time-additive utility from intermediate consumption and the aggregate dividend following a geometric Brownian motion. Basak and Cuoco (1998) study equilibria with two agents and limited stock market participation. Bhamra and Uppal (2009) also consider an economy with two agents and derive conditions under which market volatility is higher than dividend volatility. ${ }^{3}$ Cvitanić and Malamud (2009a, 2009b) study asymptotic equilibrium dynamics with an arbitrary number of CRRA agents maximizing utility from terminal consumption as the horizon increases.

Only a few papers have studied general properties of equilibria with non-CRRA preferences and/or a general dividend process. ${ }^{4}$ Our paper is partially related to He and Leland (1993). ${ }^{5}$ They also allow for an arbitrary dividend process and describe the set of viable price processes, that is, all price processes that can be attained by varying the utility of the representative agent. One of their main results declares that when the dividend is a geometric Brownian motion, MPR is countercyclical if and only if the risk aversion is countercyclical. Our representation allows us to prove the most general result of this kind. More precisely, we show that countercyclical risk aversion generally is not sufficient, and countercyclicality of dividend volatility and procyclicality of the rate of macroeconomic fluctuation are needed.

\footnotetext{
${ }^{3}$ The main message of Bhamra and Uppal (2009) is that allowing the agents to trade in an additional derivative security, making the market complete, may actually increase market volatility. Because of completeness, their equilibrium coincides with the Arrow-Debreu equilibrium of Wang (1996).

${ }^{4}$ Some papers study static, one-period economies with heterogeneous preferences. See Benninga and Mayshar (2000), Gollier (2001), Hara, Huang and Kuzmics (2007). There are also conditions for zero equilibrium trading volume. See Berrada, Hugonnier and Rindisbacher (2007).

${ }^{5}$ See also Bick (1990) and Wang (1993).
} 
In a number of models, heterogeneity arises from beliefs and asymmetric information, as in Basak (2005), Jouini and Napp (2009), Biais, Bossaerts, and Spatt (2009) and Dumas, Kurshev, and Uppal (2009). In particular, the latter paper studies excess volatility and nonmyopic optimal portfolios in a model with two CRRA agents with identical preferences but heterogeneous beliefs. Methodologically, our paper is close to Detemple and Zapatero (1991), Detemple, Garcia, and Rindisbacher (2003), Detemple and Rindisbacher (2009), Dumas, Kurshev, and Uppal (2009), and Bharma and Uppal (2009), as we use Malliavin calculus to obtain the main results.

The paper is organized as follows. Section 2 describes the setup and notation. In Section 3, we introduce the rate of macroeconomic fluctuation and in Section 4 we use the latter to derive representations for the MPR. Section 5 studies monotonicity of optimal portfolios in risk aversion. Section 6 is devoted to the properties of hedging demand. Sections 7 and 8 study a general continuous-time CAPM with only terminal consumption. Section 9 concludes.

\section{Setup and Notation}

\subsection{The Model}

The economy has a finite horizon and evolves in continuous time. Uncertainty is described by a one-dimensional, standard Brownian motion $B_{t}, t \in[0, T]$ on a complete probability space $\left(\Omega, \mathcal{F}_{T}, P\right)$, where $\mathcal{F}$ is the augmented filtration generated by $B_{t}$. There is a single state variable $D_{t}$ evolving according to

$$
D_{t}^{-1} d D_{t}=\mu^{D}\left(D_{t}\right) d t+\sigma^{D}\left(D_{t}\right) d B_{t}
$$

This diffusion process lives on $(0,+\infty)$ and $\sigma^{D}\left(D_{t}\right)>0 .{ }^{6}$

We make the following key assumption throughout the paper:

Assumption 2.1 The market is viable; that is, the unique state price density $\xi_{T}$ is given by $\xi_{T}=g\left(D_{T}\right)$ for some smooth and strictly decreasing function $g$, and the interest rate process $r_{t}$ is given by $r_{t}=r\left(D_{t}\right)$ for some smooth function $r$.

Assumption 2.1 is natural because it holds in any general equilibrium model with the single state variable $D_{t}$. Indeed, in Merton's (1973) intertemporal capital asset pricing

\footnotetext{
${ }^{6}$ We assume that $\mu^{D}$ and $\sigma^{D}$ are such that a unique strong solution exists and also that $\mu^{D} \in C^{1}\left(\mathbb{R}_{+}\right)$, $\sigma^{D} \in C^{2}\left(\mathbb{R}_{+}\right)$. In general, whenever we use a derivative of a function, we implicitly assume it exists.
} 
model (ICCAPM), $\xi_{t}=e^{-\rho t} U^{\prime}\left(D_{t}\right)$, where $D_{t}$ is the aggregate dividend and $\rho$ and $U$ are respectively the discount rate and the utility function of the representative agent, maximizing utility from intermediate consumption. Furthermore, in this case $r_{t}=r\left(D_{t}\right)$ with

$$
r(x)=\rho+\gamma^{U}(x) \mu^{D}(x)-0.5 \gamma^{U}(x) P^{U}(x)\left(\sigma^{D}(x)\right)^{2} .
$$

For the case of terminal consumption CAPM (see Bick (1990), He and Leland (1993), and Section 8 below), $\xi_{T}=U^{\prime}\left(D_{T}\right)$, where $U$ is the utility function of the representative agent. $^{7}$ In this case, the interest rate process is not determined in equilibrium and can be specified exogenously because agents need not substitute consumption between periods. For this reason, everywhere in the sequel, we will write $\xi_{T}=U^{\prime}\left(D_{T}\right)$ where we have defined $U(c)=\int_{1}^{c} g(x) d x$.

Given the interest rate process $r_{t}$, the unique state price density process $\xi=\left(\xi_{t}\right)$ is given by

$$
\xi_{t}=e^{-\int_{0}^{t} r_{s} d s} M_{t},
$$

where $M_{t}$ is the density process of the equivalent martingale measure $Q$,

$$
\left(\frac{d Q}{d P}\right)_{t}=M_{t}=E_{t}\left[M_{T}\right]
$$

Given a state price density process, we can always construct a corresponding financial market by assuming there are two types of assets available for trading at any moment: an instantaneously risk-free bond with rate of return $r_{t}$ and a risky asset, a stock, with the price process $S_{t}$ given by either

$$
S_{t}=M_{t}^{-1} E_{t}\left[M_{T} D_{T}\right]=E_{t}^{Q}\left[e^{-\int_{t}^{T} r_{s} d s} D_{T}\right]
$$

or

$$
S_{t}=M_{t}^{-1} E_{t}\left[\int_{t}^{\infty} M_{\tau} D_{\tau} d \tau\right]=E_{t}^{Q}\left[\int_{t}^{\infty} e^{-\int_{t}^{\tau} r_{\theta} d \theta} D_{\tau} d \tau\right] .
$$

The instantaneous drift and volatility of stock price $S_{t}$ are denoted by $\mu_{t}^{S}$ and $\sigma_{t}^{S}$

\footnotetext{
${ }^{7}$ In general, we could consider an economy populated by two types of agents: those deriving utility from intermediate consumption with a possibly infinite time horizon and those deriving utility from terminal consumption (wealth) at some prescribed time horizon. For example, we could think of these agents as fund managers. See Grossman and Zhou (1996) and Cuoco and Kaniel (2010). In this case, we would need to assume that the aggregate dividend has both a continuous rate component and a lumpy component, paid at those prescribed time periods.
} 
respectively, so that ${ }^{8}$

$$
\frac{d S_{t}}{S_{t}}=\mu_{t}^{S} d t+\sigma_{t}^{S} d B_{t}
$$

For simplicity, we always assume that stock volatility $\sigma_{t}^{S}$ is almost surely nonnegative. ${ }^{9}$

The MPR $\lambda_{t}$ is given by

$$
\lambda_{t}=\frac{\mu_{t}^{S}-r_{t}}{\sigma_{t}^{S}}
$$

It also can be calculated directly from the state-price density process using the equation

$$
\xi_{t}^{-1} d \xi_{t}=-r_{t} d t-\lambda_{t} d B_{t}
$$

An economic agent $k$ chooses portfolio strategy $\pi_{k t}$, the portfolio weight in the risky asset at time $t$, as to maximize the expected utility ${ }^{10}$

$$
E\left[u_{k}\left(W_{k T}\right)\right]
$$

of the terminal wealth $W_{k T}$, where the wealth $W_{k t}$ of agent $k$ evolves as

$$
d W_{k t}=W_{k t}\left[r_{t} d t+\pi_{k t}\left(S_{t}^{-1} d S_{t}-r_{t} d t\right)\right]
$$

Introduce the inverse of the marginal utility

$$
I_{k}(x):=\left(u_{k}^{\prime}\right)^{-1}(x)
$$

It is well known that in this complete market setting the optimal terminal wealth is given by

$$
W_{k T}=I_{k}\left(y_{k} \xi_{T}\right)
$$

where $y_{k}$ is determined via the budget constraint ${ }^{11}$

$$
E\left[I_{k}\left(y_{k} \xi_{T}\right) \xi_{T}\right]=W_{k 0}
$$

\section{Rate of Macroeconomic Fluctuations}

In this section we introduce a new object, the rate of macroeconomic fluctuation. This rate will play a crucial role in the sequel as it will appear in expressions for all quantities

\footnotetext{
${ }^{8}$ It is possible to show that the market is viable if and only if the diffusion coefficients of $S_{t}$ satisfy certain differential equations. See Bick (1990) and Wang (1993).

${ }^{9}$ This is always true if the interest rate $r_{t}$ is not excessively procyclical. See Appendix.

${ }^{10}$ We assume that $u_{k}$ is $C^{3}\left(\mathbb{R}_{+}\right)$and satisfies standard Inada conditions.

${ }^{11}$ We assume that a unique value of $y_{k}$ satisfying the budget constraint exists.
} 
in both partial and general equilibrium (MPR, stock volatility and optimal portfolios) and will have a fundamental impact on their static and dynamic properties.

Two forces drive the stochastic dynamics of the state variable $D_{t}$ : stochastic volatility $\sigma^{D}\left(D_{t}\right)$ and stochastic drift $\mu^{D}\left(D_{t}\right)$. The volatility $\sigma^{D}$ determines the size of macroeconomic fluctuation. To understand the role of $\mu^{D}$, we will "extract" all stochastic volatility from $D_{t}$.

Fix an $x_{0} \in \mathbb{R}_{+}$and introduce the function

$$
F(x) \stackrel{\text { def }}{=} \int_{x_{0}}^{x} \frac{1}{y \sigma^{D}(y)} d y, x>0
$$

Then a direct calculation shows that the diffusion coefficient of the process

$$
A_{t} \stackrel{\text { def }}{=} F\left(D_{t}\right)
$$

is equal to 1 . Since $A_{t}$ is also an Ito diffusion, its dynamics are given by

$$
d A_{t}=C\left(A_{t}\right) d t+d B_{t}
$$

Definition 3.1 The negative instantaneous expected change of the volatility-extracted process $A_{t}$ will be referred to as the rate of macroeconomic fluctuation and denoted $\rho_{t}$. More precisely, we define

$$
\rho_{t} \stackrel{\text { def }}{=}-C^{\prime}\left(A_{t}\right)
$$

By definition, we have

$$
\rho_{t}=c^{D}\left(D_{t}\right)
$$

with

$$
c(x)=c^{D}(x) \stackrel{\text { def }}{=}-C^{\prime}(F(x)) .
$$

It follows directly from the definition that the function $c$ is invariant under functional transformations. That is, if $D_{t}=g\left(\tilde{D}_{t}\right)$ for some process $\tilde{D}_{t}$ and a smooth, increasing function $g$, then

$$
c^{D}(g(x))=c^{\tilde{D}}(x) .
$$

In particular, the following is true

Corollary 3.2 The rate of macroeconomic fluctuation $\rho_{t}$ is constant if and only if the state variable $D_{t}$ is a smooth and strictly increasing function of an autoregressive process

$$
d A_{t}=\left(a-b A_{t}\right) d t+d B_{t} .
$$

In this case, $\rho_{t}=b$. 
As our benchmark example throughout the paper, we consider a simple generalization of Geometric Brownian Motion (GBM) as the dividend process by having $A_{t}=\log D_{t}$ satisfy (6). Then if $b>0$, the growth rate of the economy (the log dividend) is mean reverting. On the other hand, if $b<0$, the growth rate of the economy departs from the "mean" $a$ at the rate equal to $-b$.

Using a direct calculation based on Ito's formula, it is possible to verify that function $c(x)$ is given by ${ }^{12}$

$$
c(x)=-x\left(\mu^{D}\right)^{\prime}(x)+x\left(\sigma^{D}\right)^{\prime}(x) \sigma^{D}(x)^{-1} \mu^{D}(x)+\left(\sigma^{D}\right)^{\prime}(x) \sigma^{D}(x) x+0.5\left(\sigma^{D}\right)^{\prime \prime}(x) x^{2} \sigma^{D}(x) .
$$

This expression was introduced in the fundamental work of Detemple, Garcia, and Rindisbacher (2003), who used it to derive representations for optimal portfolios in general diffusion-driven models. Our new formula (5) provides a very intuitive economic interpretation of this object and makes some of its properties (such as functional invariance) particularly clear.

\section{The Market Price of Risk}

Recall that by assumption the market is viable and the state price density $\xi_{T}$ is given by $\xi_{T}=U^{\prime}\left(D_{T}\right)$ for some strictly concave function $U$. Let

$$
\gamma^{U}(x)=-\frac{x U^{\prime \prime}(x)}{U^{\prime}(x)}
$$

In an equilibrium setting, ${ }^{13} U$ is the utility of the representative agent and $\gamma^{U}$ is the relative risk aversion of the representative agent. We also will need the following:

Definition 4.1 We will refer to

$$
\sigma^{D}(t, \tau) \stackrel{\text { def }}{=} e^{-\int_{t}^{\tau} \rho_{s} d s} \sigma^{D}\left(D_{\tau}\right)
$$

as the discounted aggregate volatility. We will refer to

$$
\sigma^{r}(t, \tau) \stackrel{\text { def }}{=} e^{-\int_{t}^{\tau} \rho_{s} d s} \sigma^{r}\left(D_{\tau}\right)
$$

as the discounted interest rate volatility. We will refer to

$$
\lambda(t, \tau) \stackrel{\text { def }}{=} e^{-\int_{t}^{\tau} \rho_{s} d s} \lambda_{\tau}
$$

\footnotetext{
${ }^{12}$ See Appendix.

${ }^{13}$ Either with terminal or with intermediate consumption.
} 
as the discounted MPR, and we will refer to

$$
\sigma_{t}^{\text {myopic }} \stackrel{\text { def }}{=} E_{t}^{Q}\left[\sigma^{D}(t, T)\right]
$$

as the myopic volatility.

In general, we call myopic all quantities that are determined by the present market value of their (possibly discounted) end-of-horizon values. In other words, their current value does not depend on their future co-movement with other market variables but only on the "myopic estimate" of their future value.

The next result provides a representation of the MPR in terms of the quantities introduced in Definition 4.1 and highlights the role of myopic volatility.

Theorem 4.2 The MPR is given by the present market value of future discounted MPR net of the present cumulative value of the future discounted interest rate volatility,

$$
\lambda_{t}=E_{t}^{Q}[\lambda(t, T)]-E_{t}^{Q}\left[\int_{t}^{T} \sigma^{r}(t, s) d s\right]
$$

where

$$
\lambda_{T}=\gamma^{U}\left(D_{T}\right) \sigma^{D}\left(D_{T}\right) .
$$

In particular, if $\gamma^{U}=\gamma$ is constant, then

$$
\lambda_{t}=\gamma \sigma_{t}^{\text {myopic }}-E_{t}^{Q}\left[\int_{t}^{T} \sigma^{r}(t, s) d s\right] .
$$

Theorem 4.2 shows that the assumed market viability leads to very interesting properties of the MPR dynamics. To gain economic intuition behind the representation (9), it is instructive to compare the result of Theorem 4.2 with the analogous results for a terminal consumption CAPM. When the interest rate is constant, the dividend follows a GBM with volatility $\sigma$ and the representative agent's risk aversion $\gamma^{U}=\gamma$ is constant, we have

$$
\lambda_{t}=\gamma \sigma
$$

Formula (11) shows that when the dividend is not a GBM, the role of the fundamental volatility $\sigma$ is played by the myopic volatility $\sigma_{t}^{\text {myopic }}$. The latter is given by the present market value of future dividend volatility, discounted at the rate of macroeconomic fluctuation $\rho_{t}$, and thus depends on the dynamic properties of $D_{t}$ in a nontrivial way. In 
particular, dividend volatility by itself is not enough for determining the size of the equilibrium MPR. We need to know the rate $\rho_{t}$. For example, if $\rho_{t}$ is always positive (negative), the level of fundamental volatility leads to overestimating (underestimating) the size of the MPR.

To understand the intuition behind this discounting, consider a simple modification of Merton's CAPM, for which the log-dividend follows (6) with $b>0$ and both $\gamma^{U}=\gamma$ and $r$ are constant. Then $\rho_{t}=b$ and

$$
\lambda_{t}=\gamma \sigma e^{-b(T-t)}
$$

That is, the equilibrium riskiness of the stock is given by $\sigma e^{-b(T-t)}$. On the other hand, the stock riskiness is determined by its sensitivity to changes in the state variable $D_{t}$ : If the stock price moves significantly in response to a change in the dividend, the stock is risky; if the sensitivity is small, stock price fluctuations are small and the stock is less risky. As recalled in (2), the stock price is the expectation (under the risk-neutral measure) of future dividends. If $b>0, \log$ dividends are mean reverting, and therefore, stock price sensitivity to dividend changes is smaller than in the case $b=0$. This drives down the stock riskiness and the equilibrium MPR. However, as time $t$ approaches terminal horizon $T$, insufficient time remains for the dividends to mean-revert, and the sensitivity of expected future dividends to changes in the current value of the dividend grows exponentially at the rate $b$, equal to the rate of mean-reversion. Thus, stock riskiness also grows exponentially, and converges to the riskiness of the dividend when $t \uparrow T$.

The situation is opposite when the rate $\rho_{t}=b$ is negative. Then the log-dividend is nonstationary, and every small fluctuation around the mean may force the dividend to deviate substantially. Hence, stock is riskier than in the case $b=0$, and the MPR goes up. However, as time $t$ approaches $T$, there is insufficient time remaining for the dividend to deviate, riskiness decreases at the rate $b$, and the equilibrium MPR goes down.

When the interest rate is stochastic, another term, $E_{t}^{Q}\left[\int_{t}^{T} \sigma^{r}(t, s) d s\right]$, appears in (11). To understand the intuition behind this term, suppose that the interest rate is procyclical. ${ }^{14}$ Then bond prices are countercyclical. Thus, bonds are expensive when stocks are cheap. This drives up the demand for stocks, pushing up the equilibrium stock price, and, consequently, equilibrium stock returns and the MPR decrease.

\footnotetext{
${ }^{14}$ We call an economic variable countercyclical if it is monotonically decreasing in $D_{t}$ and procyclical otherwise.
} 
In Merton's (1973) ICCAPM, the MPR process is given by $\gamma^{U}\left(D_{t}\right) \sigma\left(D_{t}\right)$ for the representative agent's utility $U$ and the interest rate is given by (1). This specific form of the interest rate dynamics leads to the following interesting identity: in Merton's ICCAPM, we always have

$$
E_{t}^{Q}[\lambda(t, T)]-E_{t}^{Q}\left[\int_{t}^{T} \sigma^{r}(t, s) d s\right]=\gamma^{U}\left(D_{t}\right) \sigma\left(D_{t}\right)
$$

for any $T \geq t$. To the best of our knowledge, identity (12) has never previously appeared in the literature.

The following corollary is a direct consequence of Theorem 4.2.

\section{Corollary 4.3 The process}

$$
e^{-\int_{0}^{t} \rho_{s} d s} \lambda_{t}+\int_{0}^{t} e^{-\int_{0}^{s} \rho_{\theta} d \theta} \sigma_{s}^{r} d s
$$

is a martingale under the equilibrium risk-neutral measure. In particular, if the interest rate is constant, the drift of the MPR is independent of $U$ and is always equal to $\rho_{t}$.

We believe that formulae (9) and (12) and the result of Corollary 4.3 are of fundamental importance because they are model-independent. Therefore, they generate universal empirical predictions that can be used to test various versions of complete market CAPM.

For example, if we ignore the interest rate volatility term, as is justified by its very low empirical values, we get that MPR evolves under the physical measure as

$$
d \lambda_{t}=\lambda_{t}\left(\mu_{t}^{\lambda} d t+\sigma_{t}^{\lambda} d B_{t}\right)
$$

with the drift $\mu_{t}^{\lambda}$ given by

$$
\mu_{t}^{\lambda}=\rho_{t}+\lambda_{t} \sigma_{t}^{\lambda}
$$

If we estimate the MPR $\lambda_{t}$, its volatility $\sigma_{t}^{\lambda}$ and drift $\mu_{t}^{\lambda}$ from the data, we immediately obtain an estimate for the rate $\rho_{t}$ :

$$
\rho_{t}=\lambda_{t} \sigma_{t}^{\lambda}-\mu_{t}^{\lambda}
$$

In this sense, the rate of macroeconomic fluctuation is directly observable and various statistical hypotheses about $\rho_{t}$ can be directly tested. 


\section{Optimal Portfolios}

Let

$$
U_{k t}(x) \stackrel{\text { def }}{=} \sup _{\pi} E_{t}\left[u_{k}\left(W_{k T}\right) \mid W_{k t}=x\right]
$$

be the value function of agent $k{ }^{15}$ The effective relative risk aversion of agent $k$ at time $t$ is defined via

$$
\gamma_{k t}=\gamma_{k t}\left(W_{k t}\right) \stackrel{\text { def }}{=}-\frac{W_{k t} U_{k t}^{\prime \prime}\left(W_{k t}\right)}{U_{k t}^{\prime}\left(W_{k t}\right)} .
$$

When the investment opportunity set is nonstochastic, the optimal portfolio is myopic, instantaneously mean-variance efficient, and is given by ${ }^{16}$

$$
\pi_{k t}^{\text {myopic }} \stackrel{\text { def }}{=} \frac{\lambda_{t}}{\gamma_{k t} \sigma_{t}^{S}} .
$$

It is known (He and Huang (1994)) that the effective absolute risk tolerance

$$
R_{k t}=\frac{W_{k t}}{\gamma_{k t}}
$$

(i.e., the absolute risk tolerance of the value function) is a discounted martingale under the risk-neutral measure. Consequently, the following result holds:

Proposition 5.1 We have

$$
W_{k t} \pi_{k t}^{\text {myopic }}=\frac{\lambda_{t}}{\sigma_{t}^{S}} R_{k t}=\frac{\lambda_{t}}{\sigma_{t}^{S}} E_{t}^{Q}\left[e^{-\int_{t}^{T} r_{s} d s} R_{k T}\right]
$$

We denote

$$
\pi_{k t}^{\text {hedging }} \stackrel{\text { def }}{=} \pi_{k t}-\pi_{k t}^{\text {myopic }}
$$

and refer to it as the hedging portfolio. This is the nonmyopic component of the optimal portfolio that the agent uses to hedge against (or, take advantage of) future fluctuations in the stochastic opportunity set.

Recall that by Definition 4.1,

$$
\lambda(t, T) \stackrel{\text { def }}{=} e^{-\int_{t}^{T} \rho_{s} d s} \lambda_{T}, \sigma^{r}(t, \tau)=e^{-\int_{t}^{\tau} \rho_{s} d s} \sigma_{\tau}^{r}
$$

are respectively the MPR and the interest rate volatility, discounted at the rate of macroeconomic fluctuation. The following result then holds:

\footnotetext{
${ }^{15}$ Note that $U_{k t}$ depends on $D_{t}$ but we suppress this dependence.

${ }^{16}$ Merton $(1969,1971)$.
} 
Theorem 5.2 We have

$$
\pi_{k t}^{\text {hedging }}=\pi_{k t}^{\mathrm{MPR} \text { hedge }}+\pi_{k t}^{\mathrm{IR} \text { hedge }}
$$

with the MPR hedging component

$$
\begin{aligned}
\pi_{k t}^{\mathrm{MPR} \text { hedge }} & =-\frac{1}{\sigma_{t}^{S} W_{k t}} \times \\
& \operatorname{Cov}_{t}^{Q}\left(\lambda(t, T), e^{-\int_{t}^{T} r_{s} d s}\left(W_{k T}-R_{k T}\right)\right) .
\end{aligned}
$$

and the interest rate (IR) hedging component

$$
\pi_{k t}^{\mathrm{IR} \text { hedge }}=\frac{1}{\sigma_{t}^{S}}\left(\frac{1}{\gamma_{k t}}-1\right) E_{t}^{Q}\left[\int_{t}^{T} \sigma^{r}(t, \tau) d \tau\right]
$$

Formulae (14) and (15) provide closed-form expressions for hedging portfolios. They show that the nature of the MPR hedge is quite different from that of the IR hedge. The latter is a product of two simple terms: one that depends on the current level of the agent's effective risk aversion, another given by the market value of the (discounted) cumulative interest rate volatility, normalized by the stock volatility. In particular, if the interest rate is procyclical, the IR hedge is positive (negative) when effective risk aversion is below (above) $1 .{ }^{17}$ Furthermore, the IR hedges of two CRRA agents differ only by a constant multiple. ${ }^{18}$

The MPR hedge is more complex. It is determined by joint fluctuations of the discounted future MPR with agent-specific characteristics: his future wealth net of his absolute risk tolerance. Suppose for simplicity that the interest rate is constant. We can then write (14) as

$$
e^{r(T-t)} \sigma_{t}^{S} W_{k t} \pi_{k t}^{\mathrm{MPR} \text { hedge }}=\underbrace{-\operatorname{Cov}_{t}^{Q}\left(\lambda(t, T), W_{k T}\right)}_{\text {wealth hedge }}+\underbrace{\operatorname{Cov}_{t}^{Q}\left(\lambda(t, T), R_{k T}\right)}_{\text {risk tolerance hedge }} .
$$

The first term on the right-hand side is the covariance of agent's wealth with the (discounted) MPR. We will refer to it as the wealth hedge. Similarly, the second term will be referred to as the risk tolerance hedge.

To understand the nature of the wealth hedge, suppose that the future MPR and the agent's wealth are positively correlated. When the agent's wealth is low, any

\footnotetext{
${ }^{17}$ Detemple, Garcia and Rindisbacher (2003) derive an analogous result in a partial equilibrium setting for a class of multi-dimensional diffusion state variables.

${ }^{18} \mathrm{By}(13), \gamma_{k t}=\gamma_{k}$ if agent $k$ has a CRRA utility. This also follows from a simple homogeneity argument.
} 
additional unit of wealth is valuable. Since MPR is also low in those states, the agent invests more in the risk-free asset because it offers a better hedge against the low-wealth states. This generates a negative hedging demand. When the agent's wealth is high, the utility of receiving an additional unit of wealth is low. therefore, even though the MPR is high in those states, it does not make the risky asset that much more attractive. ${ }^{19}$

To illustrate the intuition behind the risk tolerance hedge, suppose that the MPR and risk tolerance are positively correlated. Then when risk tolerance is high, the agent is willing to take more risk, and this effect is magnified since the MPR is high in those states, driving up the demand for stock. When risk tolerance is low, the MPR is also low. This generates an incentive to hedge against those states and to reduce the long position in the stock, but not to sell short excessively, because of low risk tolerance. As a consequence, stock investment is altogether more attractive (the impact of the states with high MPR and high risk tolerance prevails) and this generates a positive hedging demand. ${ }^{20}$

If we adopt Arrow's hypothesis that absolute risk aversion is monotonically decreasing in wealth, risk tolerance $R_{k T}=R_{k}\left(W_{k T}\right)$ is increasing in wealth and, generally speaking, the wealth hedge and the risk tolerance hedge will have opposite signs and represent competing effects for determining the size of the hedging demand. Note also that if the agent's utility is of the CARA (exponential) class, $R_{k T}$ is constant and, consequently, the risk tolerance hedge vanishes.

If agent $k$ has a HARA utility, we have

$$
R_{k T}=a W_{k T}+b
$$

for some constants $a, b$ and the MPR hedge takes the form

$$
e^{r(T-t)} \sigma_{t}^{S} W_{k t} \pi_{k t}^{\mathrm{MPR} \text { hedge }}=(a-1) \operatorname{Cov}_{t}^{Q}\left(\lambda(t, T), W_{k T}\right) .
$$

This formula generates the empirical prediction that MPR hedges are driven by the comovement of the agent's wealth with the discounted MPR. This prediction can be tested using PSID data (see Brunnermeier and Nagel 2008).

One also could use this formula together with the empirical state price density method of Rosenberg and Engle (2002) to estimate the empirical optimal hedging portfolio.

\footnotetext{
${ }^{19}$ The argument for the case of negative correlation between wealth and the MPR is analogous.

${ }^{20}$ The case in which the MPR and risk tolerance are negatively correlated is analogous.
} 


\section{Monotonicity of Optimal Portfolios in Risk Aversion}

A cornerstone of the Arrow-Pratt theory of risk aversion is that in the standard oneperiod portfolio allocation problem with one risky and one risk-free asset, the fraction of wealth invested in the risky asset is monotonically decreasing in risk aversion. ${ }^{21}$

Monotonicity of optimal portfolios in risk aversion is also crucial for understanding the nature of risk-sharing in dynamic general equilibrium models. In fact, almost all existing papers on heterogeneous equilibria conjecture this monotonicity property and use it as the basis for their economic intuition, arguing that more-risk-averse agents will hold less stock and lend money to the less-risk-averse ones. (See Dumas 1989, Wang 1996, Basak and Cuoco 1998, Basak 2005, Bhamra and Uppal 2009). It is therefore surprising that over the past 30 years, there has been no progress in extending the ArrowPratt monotonicity result to a multi-period setting, neither in partial nor in general equilibrium. The goal of this section is to address this deficiency. We obtain strong sufficient conditions for the monotonicity of optimal portfolios in risk aversion that are generally applicable and, we believe, are tight.

Of course, one cannot expect the Arrow-Pratt monotonicity result to hold in a general multi-period setting. Even in the case when returns are independent over time and the portfolio choice problem is myopic, stochastic future returns have a "background risk" effect on the agent's value function. ${ }^{22}$ In particular, an ordering of risk aversions $\gamma_{i}$ and $\gamma_{j}$ by no means implies the same ordering of the effective risk aversions $\gamma_{i t}$ and $\gamma_{j t}$. In order to formulate conditions guaranteeing this ordering of risk aversions, we will need a definition due to Ross (1981).

Definition 6.1 (Ross (1981)) For agent $i$, we denote

$$
\gamma_{i}^{\inf } \stackrel{\text { def }}{=} \inf _{x} \gamma_{i}(x), \gamma_{i}^{\text {sup }} \stackrel{\text { def }}{=} \sup _{x} \gamma_{i}(x)
$$

Agent $i$ is more risk averse than agent $j$ in the sense of Ross, if

$$
\gamma_{i}^{\text {inf }} \geq \gamma_{j}^{\text {sup }}
$$

In this case we write $\gamma_{i} \geq_{R} \gamma_{j}$.

\footnotetext{
${ }^{21}$ See Pratt (1964) and Arrow (1965).

${ }^{22}$ Pratt and Zeckhauser (1987), Gollier and Pratt (1995) and Gollier and Zeckhauser (2002).
} 
Now, by (13),

$$
\gamma_{i t}=\frac{W_{i t}}{E_{t}^{Q}\left[e^{-\int_{t}^{T} r_{s} d s} \gamma_{i T}^{-1} W_{i T}\right]}=\frac{E_{t}^{Q}\left[e^{-\int_{t}^{T} r_{s} d s} W_{i T}\right]}{E_{t}^{Q}\left[e^{-\int_{t}^{T} r_{s} d s} \gamma_{i T}^{-1} W_{i T}\right]} \in\left[\gamma_{i}^{\text {inf }}, \gamma_{i}^{\text {sup }}\right] .
$$

We arrive at

Proposition 6.2 If $\gamma_{i} \geq_{R} \gamma_{j}$ then $\gamma_{i t} \geq \gamma_{j t}$.

Thus, when stock returns are independent, the ordering of risk aversions in the sense of Ross implies the corresponding ordering of optimal myopic portfolio weights. However, when the investment opportunity set is stochastic, optimal portfolios are nonmyopic and contain a highly nontrivial hedging component, whose dependence on risk aversion is by no means obvious. The presence of this hedging demand may completely change portfolio behavior. ${ }^{23}$ It is therefore crucial to identify conditions concerning the nature of stochastic fluctuations in the investment opportunity under which the natural risk-taking behavior is preserved. There are three fundamental sources of these stochastic fluctuations: stochastic volatility $\sigma_{t}^{D}$, stochastic interest rates $r_{t}$, and the stochastic rate of macroeconomic fluctuation $\rho_{t}$. When $\rho_{t}$ is constant, it turns out that optimal portfolio weights are always monotonically decreasing in risk aversion. This follows from the following theorem.

Theorem 6.3 Suppose that the rate of macroeconomic fluctuation $\rho_{t}$ is constant. Then optimal portfolio weights are monotonically decreasing in risk aversion in the sense of Ross.

The result of Theorem 6.3 is quite general, as it imposes absolutely no constraints on the nature of the agent's utility function, MPR $\lambda_{T}$, and dividend volatility $\sigma\left(D_{T}\right)$. If the MPR $\lambda_{T}$ is stochastic, Theorem 5.2 implies that optimal portfolios have nonmyopic components that are not necessarily monotonic in risk aversion. Nevertheless, the monotonicity of the myopic component fully compensates for the possible nonmonotonicity of the nonmyopic components.

Another important case in which the monotonicity result holds is when risk aversions of the two agents are on different sides of 1 . Using the fact that the log agent is myopic and always holds an instantaneously mean-variance efficient portfolio $\pi_{\log t}=$ $\left(\sigma_{t}^{S}\right)^{-1} \lambda_{t}$, it is possible to show that the following is true. ${ }^{24}$

\footnotetext{
${ }^{23}$ A simple Example B.3 in the Appendix shows that nonmyopic portfolios are generally not monotonic in risk aversion even in simple two-period binomial models.

${ }^{24}$ The proof follows directly from Proposition B.1 in the appendix.
} 
Proposition 6.4 Suppose that $\gamma_{i} \leq_{R} 1 \leq_{R} \gamma_{j}$. Then

$$
\pi_{i t} \geq \pi_{\log t} \geq \pi_{j t}
$$

Thus, when risk aversions are on different sides of 1 , the monotonicity result holds independently of the model parameters. However, when risk aversions are both on the same side of 1 , and the rate of macroeconomic fluctuation $\rho_{t}$ is stochastic, stronger conditions about the joint variation of the rate of macroeconomic fluctuation and the $\operatorname{MPR} \lambda_{T}$ need to be imposed, as in the following theorem.

Theorem 6.5 Suppose that one of the following is true:

(i) $\gamma_{i} \geq_{R} \gamma_{j} \geq 1$ and both $-\rho_{t}$ and $\lambda_{T}$ are countercyclical;

(ii) $1 \geq \gamma_{i} \geq_{R} \gamma_{j}$ and both $-\rho_{t}$ and $\lambda_{T}$ are procyclical.

Then agent i's portfolio weight is smaller than agent $j$ 's.

To gain intuition about how optimal portfolio behavior depends on the joint dynamics of $\rho_{t}$ and $\lambda_{T}$, recall that by (16), the nonmyopic value of the stock investment for an agent is determined by the future co-movement of the MPR with the agent's wealth and risk tolerance. If agent $i^{\prime} s$ wealth net of his risk tolerance (i.e., $W_{i T}-R_{i T}$ ) co-moves more strongly with the future market price of risk than that of agent $j$, he will hold a larger fraction of wealth in the nonmyopic portfolio. The latter may happen even if agent $i$ is more risk-averse than agent $j$. Thus, there will be two competing effects, determining the total size of investment and it is a priori unclear which effect dominates. Suppose for simplicity that both agents $k=i, j$ are CRRA agents with $\gamma_{i}>\gamma_{j}>1$. Introduce an agent-specific probability measure ${ }^{25}$

$$
d Q^{k} \stackrel{\text { def }}{=} \frac{e^{-\int_{t}^{T} r\left(D_{s}\right) d s} W_{k T}}{E^{Q}\left[e^{-\int_{t}^{T} r\left(D_{s}\right) d s} W_{k T}\right]} d Q
$$

By construction, this measure assigns weights to various states of the world according to the size of the agent's discounted wealth $e^{-\int_{t}^{T} r\left(D_{s}\right) d s} W_{k T}$ in those states. Using Theorem 5.2 , it is possible to show that

$$
\sigma_{t}^{S} \pi_{k t}=-\left(1-\gamma_{k}^{-1}\right) E_{t}^{Q_{k}}[\lambda(t, T)]+\lambda_{t} .
$$

\footnotetext{
${ }^{25}$ Cvitanić and Malamud (2010) call this measure the agent $k$ 's "wealth forward measure".
} 
Thus, the size of agent $i$ 's optimal portfolio is driven by two effects: the size of his risk aversion $\gamma_{k}$ and the size of the expectation of the discounted $\operatorname{MPR} \lambda(t, T)$ under the agent-specific measure $Q^{k}$. This expectation precisely captures the nonmyopic nature of the portfolio weight $\pi_{k t}$. Since measure $Q^{k}$ assigns larger weight to those states in which agent $i^{\prime} s$ wealth is large, it will be larger for an agent whose wealth more strongly co-varies with the discounted market price of risk. Since the markets are complete, wealth $W_{k T}=I_{k}\left(y_{k} \xi_{T}\right)=\left(y_{k} U^{\prime}\left(D_{T}\right)\right)^{-\gamma_{k}^{-1}}$ is monotonically increasing in $D_{T}$, and the sensitivity of $W_{k T}$ to changes in $D_{T}$ is determined by the risk tolerance $\gamma_{k}^{-1}$. Thus, when $\gamma_{k}$ is low, the wealth $W_{k T}$ will be very sensitive to changes in $D_{T}$ and, consequently, is highly procyclical. Therefore, agent $k$ will bet on the realization of states with very high $D_{T}$ and the measure $Q^{k}$ will put great weight on those states. For this reason, if $\lambda(t, T)$ is countercyclical, its expectation $E^{Q^{k}}[\lambda(t, T)]$ is monotonically increasing in $\gamma_{k}{ }^{26}$ and therefore

$$
\frac{1-\gamma_{i}^{-1}}{1-\gamma_{j}^{-1}}>1>\frac{E_{t}^{Q_{j}}[\lambda(t, T)]}{E_{t}^{Q_{i}}[\lambda(t, T)]} .
$$

The claim of Theorem 6.5 follows then from (18) and (17).

By contrast, when $\lambda(t, T)$ is procyclical, its expectation $E^{Q^{k}}[\lambda(t, T)]$ is monotonically decreasing in $\gamma_{k}$ because agents with low risk aversion bet on the realization of states with high $D_{T}$ and, consequently, high $\lambda(t, T)$.

\section{The Sign of the Hedging Demand}

As discussed above, the exact behavior of nonmyopic optimal portfolios depends on the cyclicality of the rate of macroeconomic fluctuation $\rho_{t}$ and the MPR $\lambda_{T}$. In this section we provide necessary and sufficient conditions for the positivity of MPR hedging demand, depending only on the properties of the agent's utility function and cyclicality of $\rho_{t}$ and $\lambda_{T}$.

Recall that the absolute prudence and absolute risk tolerance of agent $k$ are defined as

$$
P_{k}(x)=-\frac{u_{k}^{\prime \prime \prime}(x)}{u_{k}^{\prime \prime}(x)} \quad \text { and } \quad R_{k}(x)=-\frac{u_{k}^{\prime}(x)}{u_{k}^{\prime \prime}(x)}
$$

respectively. The following is true:

Theorem 7.1 Suppose that both $-\rho_{t}$ and $\lambda_{T}$ are countercyclical (procyclical) and that $r^{\prime}$ is not too large. Then the MPR hedging component $\pi_{t}^{\mathrm{MPR}}$ hedge is positive if and only

\footnotetext{
${ }^{26}$ This heuristic argument is made rigorous in the proof of Theorem 6.5 in the Appendix.
} 
if the product of prudence and risk tolerance is below (above) $2^{27}$, that is

$$
P_{k}(x) R_{k}(x) \leq 2 \quad \text { for all } x
$$

The result of Theorem 7.1 is somewhat unexpected at first glance. Since the optimal portfolio of a log investor is always myopic, one would expect that the sign of the hedging component depends only on whether risk aversion is above or below 1 (as it does for the IR hedging component). However, Theorem 7.1 shows that the hedging motive depends on properties of three derivatives of the utility function.

To understand the economic mechanisms behind Theorem 7.1, consider the case when both $-\rho_{t}$ and $\lambda_{T}$ are countercyclical and suppose for simplicity that $r_{t}=r$ is constant. Recall the expression (16):

$$
e^{r(T-t)} \sigma_{t}^{S} W_{k t} \pi_{k t}^{\mathrm{MPR} \text { hedge }}=-\operatorname{Cov}_{t}^{Q}\left(\lambda(t, T),\left(W_{k T}-R_{k T}\right)\right)
$$

Since markets are complete, $W_{k T}$ is increasing in $D_{T}$ and, consequently, the wealth hedge $-\operatorname{Cov}_{t}^{Q}\left(\lambda(t, T), W_{k T}\right)$ is positive. The size of the risk tolerance hedge depends on the cyclical properties of the agent's risk tolerance $R_{k T}$. A direct calculation shows that

$$
\frac{d}{d x} R_{k}(x)=-1+P_{k}(x) R_{k}(x)
$$

If the product $P_{k} R_{k}$ is large, the risk tolerance $R_{k T}$ is highly sensitive to wealth fluctuations and, consequently, is highly procyclical. Then the risk tolerance hedge

$$
\operatorname{Cov}_{t}^{Q}\left(\lambda(t, T), R_{k T}\right)
$$

is very negative and dominates the wealth hedge,

$$
-\operatorname{Cov}_{t}^{Q}\left(\lambda(t, T), W_{k T}\right)
$$

and the MPR hedge is negative. By contrast, if $P_{k} R_{k}$ is small, the risk tolerance hedge is not very negative. Therefore, the wealth hedge dominates and the MPR hedge is positive. The threshold of 2 appears because $W_{k T}-R_{k}\left(W_{k T}\right)$ is monotonically increasing in $W_{k T}$ if and only if $P_{k} R_{k} \leq 2$.

The assumption of countercyclical MPR is natural and strong empirical evidence supports it (See Campbell and Cochrane 1999). Furthermore, the countercyclical MPR

\footnotetext{
${ }^{27}$ Note that the product of prudence and risk tolerance is exactly 2 for the log investor, for whom the hedging portfolio is zero.
} 
can be generated from simple microeconomic assumptions about individual agents' preferences. ${ }^{28}$ Since this study is the first to introduce the rate of macroeconomic fluctuation, there are no empirical data about the behavior of $\rho_{t}$. We believe this is an important question deserving a serious empirical analysis. Note also that, in the benchmark case when $\rho_{t}$ is constant (see Corollary 3.2), this issue does not arise.

The appearance of prudence is also related to precautionary savings. As Kimball (1990) showed in a static, one-period model, the strength of the precautionary savings motive for an agent anticipating stochastic fluctuations in future income is determined by his prudence $P_{k}$. Here, $P_{k}$ plays a similar role, determining the strength of the savings/investment motive for an agent who anticipates future changes in the stochastic investment opportunity set.

If we consider the Arrow (1965) hypothesis that $\gamma_{k}(x)$ is increasing, a direct calculation shows that it holds if and only if $x P_{k}(x) \leq \gamma_{k}(x)+1$. If $\gamma_{k}(x) \geq 1$, we get $P_{k}(x) R_{k}(x)=x P_{k}(x) / \gamma_{k}(x) \leq 2$. Theorem 7.1 then implies the following

Corollary 7.2 Let $-\rho_{t}$ and $\lambda_{T}$ be countercyclical. Suppose that $\gamma_{k}(x) \geq 1$ and is increasing and $r^{\prime}$ is not excessively large. Then the MPR hedge is positive.

For the benchmark CRRA utility, $P_{k} R_{k}=1+\gamma_{k}^{-1}$ and the results take a simpler form

Corollary 7.3 Suppose that $\gamma_{k}=$ const, $r^{\prime}$ is not excessively large, and both $-\rho_{t}$ and $\lambda_{T}$ are countercyclical. Then the MPR hedge is positive if and only if $\gamma_{k} \geq 1$.

The intuition behind Corollary 7.3 is as follows. The marginal utility $u_{k}^{\prime}(x)=x^{-\gamma_{k}}$ of an agent with high risk aversion $\gamma_{k}>1$ is high in bad states (low $D_{t}$ ). Therefore, any additional unit of consumption in those states is highly valuable for him. Since the MPR is countercyclical, it is high in bad states. This makes the stock a highly attractive instrument for agent $k$ to hedge against those states and induces him to buy additional shares. On the other hand, an agent with low risk aversion $\gamma_{k}<1$ is not "afraid" of the bad states and bets on the realization of good states with high $D_{t}$. Since the MPR is low in those states, it is optimal for him to sell some of his stock holdings, creating a negative hedging demand.

\footnotetext{
${ }^{28}$ See Proposition 9.3 below.
} 


\section{Equilibrium Dynamics}

\subsection{Setup}

In this section, we study implications of the agent's nonmyopic portfolio behavior for equilibrium dynamics. We assume that $D_{T}$ is the terminal dividend of the single risky asset in positive net supply and there are no intermediate dividends.

There are $K$ competitive agents behaving rationally. Agent $k$ is initially endowed with $\psi_{k}>0$ shares of stock, and the total supply of the stock is normalized to 1 ,

$$
\sum_{k=1}^{K} \psi_{k}=1
$$

Agent $k$ chooses portfolio strategy $\pi_{k t}$, the portfolio weight at time $t$ in the risky asset, as to maximize expected utility ${ }^{29}$

$$
E\left[u_{k}\left(W_{k T}\right)\right]
$$

of the terminal wealth $W_{k T}$. As usual, we say that the market is in equilibrium if the agents behave optimally and both the risky asset market and the risk-free market clear.

It is well known that the above financial market is dynamically complete if the volatility process $\sigma_{t}^{S}$ of the stock price is almost surely nonzero. ${ }^{30}$ Because of the market completeness, any equilibrium allocation is Pareto-efficient and can be characterized as an Arrow-Debreu equilibrium (See Duffie and Huang 1986, Wang 1996). ${ }^{31}$

Since in equilibrium the final wealth amounts of all agents must sum up to the aggregate dividend, equilibrium state price density $\xi_{T}$ is uniquely determined by the consumption market clearing equation

$$
\sum_{k=1}^{K} I_{k}\left(y_{k} \xi_{T}\right)=D_{T} .
$$

\footnotetext{
${ }^{29}$ We assume that $u_{k}$ is $C^{3}\left(\mathbb{R}_{+}\right)$and satisfies standard Inada conditions.

${ }^{30}$ This can be verified under some technical regularity conditions on the model primitives. See Hugonnier, Malamud, and Trubowitz (2009).

${ }^{31}$ Since endowments are colinear (all agents hold shares of the same single stock), it can be shown that under some conditions on the agents' utility functions, the equilibrium is in fact unique up to a multiplicative factor, and also is unique if we fix the risk-free rate. See Dana $(1995,2001)$. If the dividend is neither bounded away from zero nor from infinity, additional care is needed to verify the existence of equilibrium. See Dana (2001) and Malamud (2008). We implicitly assume throughout the paper that an equilibrium exists.
} 
It is important to mention that the model with a finite terminal horizon should be seen as an abstraction and that we should be careful interpreting it. $S_{t}$ is seen as the price of a stock whose first dividend is paid at time $T$, or at a later period. In equilibrium, $S_{T}=D_{T}$ and therefore $D_{T}$ is just the stock price at time $T$. Furthermore, $\sigma^{D}\left(D_{T}\right)$ is the local volatility and can be estimated directly from the prices of options with maturity T, using Dupire's formula (see Dumas, Fleming, and Whaley 1998). Finally, the agent's utilities $u_{k}$ from terminal wealth could be thought of as their effective utilities (value functions), derived from consumption after time $T$.

\subsection{Equilibrium Stock Price Volatility}

Unlike the MPR, the stock price volatility is a nonmyopic object. It depends not only on the size of the aggregate risk but also on its future fluctuations relative to other variables. More precisely, the following is true:

Theorem 8.1 The equilibrium stock price volatility is given by

$$
\sigma_{t}^{S}=\sigma_{t}^{\text {myopic }}+\sigma_{t}^{\text {nonmyopic }}-E_{t}^{Q}\left[\int_{t}^{T} \sigma^{r}(t, s) d s\right]
$$

where $^{32}$

$$
\sigma_{t}^{\text {nonmyopic }}=-\frac{1}{S_{t}} \operatorname{Cov}_{t}^{Q}\left(\left(\lambda(t, T)-\sigma^{D}(t, T)\right), e^{-\int_{t}^{T} r\left(D_{s}\right) d s} D_{T}\right) .
$$

Furthermore, $\sigma_{t}^{S}$ is positive if $r^{\prime}$ is sufficiently small (the interest rate is not highly procyclical).

To understand the intuition behind Theorem 8.1, we first discuss our benchmark extension of Merton's CAPM when $r, \gamma^{U}$ are constant, and the log dividend follows the autoregressive process (6). Then $\sigma^{D}$ and the rate $\rho_{t}=b$ are constant and the nonmyopic volatility $\sigma_{t}^{\text {nonmyopic }}$ vanishes. Consequently, by Theorems 4.2 and 8.1, the risk-return tradeoff coincides with that of Merton's CAPM:

$$
\lambda_{t}=\gamma \sigma_{t}^{S}
$$

However, the stock volatility does not coincide with the fundamental dividend volatility. Rather, we have

$$
\sigma^{S}=\sigma_{t}^{\text {myopic }}=e^{-b(T-t)} \sigma^{D}
$$

\footnotetext{
${ }^{32} \mathrm{We}$ use $\operatorname{Cov}_{t}^{Q}(X, Y)$ to denote conditional covariance of random variables $X$ and $Y$ under the equilibrium risk-neutral measure $Q$.
} 
Stock volatility is higher than the dividend volatility if the log dividend is nonstationary $(b<0)$ and lower if the log dividend is stationary and mean-reverting $(b>0)$, because stock price volatility is the sensitivity of expected future dividends to changes in $D_{t}$, as seen from (22) below. This sensitivity is large (small) if the log dividend is nonstationary (stationary). See also the discussion after Theorem 4.2.

If the interest rate is constant, expression (20) implies that the spread between the stock price volatility and the myopic volatility is given by the covariance of the discounted future aggregate dividend with the future discounted MPR net of the discounted future dividend volatility. The term "nonmyopic" is most easily justified if we recall that

$$
\lambda(t, T)-\sigma^{D}(t, T)=\left(\gamma^{U}\left(D_{T}\right)-1\right) \sigma^{D}(t, T)
$$

Formulae (21) and (20) imply that, generically, the nonmyopic volatility vanishes if and only if $\gamma^{U} \equiv 1$. That is, precisely when the representative agent is completely myopic. Hence, the nature of the nonmyopic volatility is necessarily driven by the agents' nonmyopic equilibrium behavior. When MPR is stochastic, nonmyopic agents increase or decrease their stock holdings depending on the expected future fluctuations of the MPR. The equilibrium hedging demand raises or decreases the total equilibrium demand for stocks and therefore drives the equilibrium stock price up or down. Since $D_{t}$ is the single state variable in our model, standard results imply that $S_{t}=S\left(t, D_{t}\right)$ is a smooth function ${ }^{33}$ of $D_{t}$, and therefore, by Ito's formula, we get

$$
\sigma_{t}^{S}=\sigma^{D}\left(D_{t}\right) D_{t} \frac{\partial}{\partial D_{t}} \log S\left(t, D_{t}\right)
$$

Thus, stock price volatility is nothing but the sensitivity of the stock price to changes in the dividend. Since equilibrium optimal portfolios respond to changes in $D_{t}$ in a nonmyopic way, so does the equilibrium stock price, giving rise to nonmyopic volatility.

Formula (20) for the nonmyopic volatility is similar to the formula for the forwardfutures spread, which says that the forward-futures spread is given by the covariance under the risk-neutral measure of the future payoff with the cumulative return on shortterm bonds (the bank account). As has been recognized in the literature, such a representation immediately generates two empirical predictions for the spread: (1) a weak prediction that the sign of the spread should coincide with the sign of the covariance, and (2) a strong prediction that the variation in the spread is explained by the covariance (see Meulbroek 1992). Based on formula (20), we can make analogous predictions for

\footnotetext{
${ }^{33}$ Under some technical conditions. See Hugonnier, Malamud, and Trubowitz (2009).
} 
the dynamics of the nonmyopic volatility and the co-movement of the dividends with the discounted MPR $\lambda(t, T)$ net of the discounted dividend volatility $\sigma^{D}(t, T)$.

There is also a clear similarity between expressions (14) and (20) for the MPR hedge and the nonmyopic volatility respectively. In fact, the hedging demand (14) is the source of nonmyopic volatility (20). Both arise as a nonmyopic response to the future fluctuations of the MPR. Similar to (20), expression (14) does not directly depend on the utility function of the representative agent. Therefore, Theorem 8.1 also has direct implications for the dynamics of the risk-return tradeoff. Empirical evidence suggests that expected stock returns are weakly related to volatility. The results of Gennotte and Marsh (1993), Glosten, Jagannathan and Runkle (1993) and Harvey (2001) suggest that the relation between expected returns and volatility is highly complex and nonlinear. One possible explanation of this phenomenon is equilibrium hedging behavior. Suppose for simplicity that the risk aversion $\gamma^{U}=\gamma$ of the representative agent and the interest rate $r$ are constant. Then by a fundamental result of Merton (1973), we have

$$
\lambda_{t}=\gamma\left(\sigma_{t}^{S}+\lambda_{t}^{\text {hedge }}\right)
$$

where $\lambda_{t}^{\text {hedge }}$ is the so-called hedge component appearing due to the equilibrium hedging

demand. Theorems 4.2 and 8.1 imply that $\lambda_{t}^{\text {hedge }}=-\sigma_{t}^{\text {nonmyopic }}$, providing a direct economic interpretation for Merton's hedge component. Furthermore, when interest rate $r$ is constant, the risk-return relation is driven by the nonlinear relation between myopic and nonmyopic volatility,

$$
\frac{\lambda_{t}}{\sigma_{t}^{S}}=\gamma \frac{\sigma_{t}^{\text {myopic }}}{\sigma_{t}^{\text {myopic }}+\sigma_{t}^{\text {nonmyopic }}} .
$$

Thus, calculating the risk-return tradeoff amounts to calculating the ratio of the nonmyopic and the myopic volatility. One can explore empirically this ratio using representations (8) and (20). It is particularly convenient that the latter does not explicitly depend on the utility of the representative agent and can be directly estimated from data using the empirical state price density (see Rosenberg and Engle 2002).

\section{Market Price of Risk Counter-cyclicality and Excess Volatil- ity}

To address further dynamic properties of equilibrium, we will need to make additional assumptions about the evolution of $D_{t}$. It has become common in the literature to use 
consumption volatility as a measure of macroeconomic uncertainty. There is empirical evidence that macroeconomic uncertainty is countercyclical (see French and Sichel 1993 and Kim et al. 2009).

Definition 9.1 We say that our model exhibits countercyclical macroeconomic uncertainty if the dividend volatility $\sigma_{t}^{D}$ is countercyclical and the rate of macroeconomic fluctuation $\rho_{t}$ is procyclical.

This definition is natural because volatility $\sigma^{D}$ and $-\rho_{t}$ determine respectively the size and the speed of macroeconomic fluctuation. From now on we make the following:

Assumption 9.2 The economy exhibits countercyclical macroeconomic uncertainty, and the aggregate risk aversion $\gamma^{U}$ is countercyclical.

By Definition 3.1, the economy exhibits countercyclical macroeconomic uncertainty if and only if $\log D_{t}=f\left(A_{t}\right)$ where $f$ is increasing and concave and the process $A_{t}$ has constant volatility and concave drift. For example, a simple family of such processes arises when

$$
d A_{t}=\left(a-b A_{t}\right) d t+\sigma d B_{t} .
$$

and the dividend process is given by $D_{t}=e^{f\left(A_{t}\right)}$ for some increasing and concave function $f$.

The assumption of countercyclical risk aversion is natural, and strong empirical evidence supports it (See Campbell and Cochrane 1999). Furthermore, countercyclical aggregate risk aversion can be generated from simple microeconomic assumptions about individual agents' preferences. In fact, it is known that if all agents in the economy have (heterogeneous) CRRA preferences the representative agent's utility will have a decreasing (i.e., countercyclical) relative risk aversion (DRRA) (see Benninga and Mayshar 2000 and Cvitanić and Malamud 2010b). A modification of the arguments from the latter paper also implies that the following slight generalization is true:

Proposition 9.3 If all agents have DRRA preferences, the aggregate risk aversion is countercyclical.

In fact, it is possible to show that Proposition 9.3 still holds if individual risk aversions are sufficiently heterogeneous and "not too increasing".

It is a conventional wisdom that countercyclical risk aversion generates countercyclical MPR. ${ }^{34}$ The following result confirms this intuition.

\footnotetext{
${ }^{34}$ See Campbell and Cochrane (1999). There is also empirical evidence supporting countercyclicality of both MPR and aggregate risk aversion. See Fama and French (1989) and Ferson and Harvey (1991).
} 
Proposition 9.4 Under Assumption 9.2, the MPR is countercyclical if both $r^{\prime}$ and $r^{\prime \prime}$ are sufficiently small. ${ }^{35}$

When the interest rate $r_{t}=r$ is constant and the dividend follows a geometric Brownian motion, He and Leland (1993) showed that the MPR is countercyclical if the aggregate risk aversion is countercyclical. Proposition 9.4 provides general sufficient conditions for the MPR countercyclicality. These conditions are tight in that a sufficiently countercyclical rate of macroeconomic fluctuation can generate procyclical behavior in MPR even if the dividend volatility is constant and risk aversion is countercyclical.

The requirement that $r^{\prime}$ and $r^{\prime \prime}$ be small is not overly restrictive, given that, empirically, interest rate volatility is very small, especially relative to stock volatility. However, from a theoretical perspective, it is interesting that the effect of stochastic interest rates on the MPR might lead to unexpected equilibrium dynamics if countercyclicality of the aggregate risk aversion is not sufficiently strong. Consider, for example, the case when $\gamma^{U}=\gamma$ is constant and the dividend is GBM. In this case the MPR is given by

$$
\lambda_{t}=\sigma\left(\gamma-E_{t}^{Q}\left[\int_{t}^{T} r^{\prime}\left(D_{\tau}\right) D_{\tau} d \tau\right]\right) .
$$

Consequently, the dynamics of the MPR are determined exclusively by the interest rate process. If $r^{\prime}\left(D_{t}\right) D_{t}$ is larger than $\gamma$ with high probability, the MPR may become negative because bond prices become so countercyclical that investors are eager to buy stock for hedging purposes even if the stock offers a negative excess return. Furthermore, if $r^{\prime}\left(D_{t}\right) D_{t}$ is decreasing in $D_{t}$, the MPR becomes procyclical.

By Proposition 9.4, countercyclical macroeconomic uncertainty together with countercyclical risk aversion generates countercyclical dynamics of MPR. The latter dynamics, in turn, determine the sign of nonmyopic volatility, as can be seen from expression (20) and the definition of $\lambda(t, T)$. More precisely, we arrive at the following result:

Proposition 9.5 Under Assumption 9.2, the nonmyopic volatility is positive if $\gamma^{U} \geq 1$ and $r^{\prime}$ is sufficiently small.

The reasoning behind this result is as follows. Under Assumption 9.2, the MPR is low in good states, but the future aggregate risk aversion is also (relatively) low and the expected future dividends are high. Therefore, the agent is willing to hold the stock

\footnotetext{
${ }^{35}$ In fact, we show that the following is true: for any interval $\left[d_{1}, d_{2}\right] \subset \mathbb{R}_{+}$there exists an $\varepsilon>0$ such that $\lambda_{t}$ is monotonically decreasing in $D_{t}$ in the interval, as long as $r^{\prime}<\varepsilon$ and $\left|r^{\prime \prime}\right|<\varepsilon$.
} 
despite low MPR. This makes the price rise in good states and, by similar arguments, decline in bad states, driving up the nonmyopic part of the price volatility. Put differently, the tension between the movements in future dividends and the MPR creates nonmyopic (excess) volatility, because the larger the dividend, the larger the demand for the stock, while at the same time the lower the MPR, the lower the demand. The requirement that $r^{\prime}$ be not excessively large is important: if the interest rate is highly procyclical, discounted dividends $e^{-\int_{t}^{T} r_{s} d s} D_{T}$ may exhibit countercyclical behavior, which, by (20), gives rise to a negative nonmyopic volatility.

The nonmyopic volatility is the natural analog of excess volatility for the case of stochastic dividend risk. ${ }^{36}$ Our representation illustrates that dynamical properties of aggregate risk aversion and dividend volatility by themselves are not sufficient to generate excess volatility and the outcome is strongly influenced by the dynamics of the rate of macroeconomic fluctuation. This result should be contrasted with a related result of Bhamra and Uppal (2009). They analyze equilibrium with two CRRA agents and a GBM dividend and show that the stock price volatility is higher than the dividend volatility if the agent's elasticity of intertemporal substitution (EIS) is not too large. Their model differs from ours because they allow for intermediate consumption, therefore, the interest rate is determined endogenously by the agents' EIS. ${ }^{37}$ Our volatility representation for the nonmyopic (excess) volatility (see Theorem 8.1) allows us to view the result of Bhamra and Uppal (2009) from a different perspective. Two sources generate deviations from fundamental (myopic) volatility in their model. Countercyclical MPR drives up the nonmyopic volatility, whereas the procyclical risk-free rate drives down the volatility, and the constraint of EIS being not too large diminishes the second effect.

\footnotetext{
${ }^{36}$ Stock prices are said to exhibit excess volatility if they are substantially more volatile than the underlying dividends. Excess volatility is a well known stylized fact. See Shiller (1981), LeRoy and Porter (1981), Mankiw, Romer, and Shapiro (1985, 1991) and West (1988).

${ }^{37}$ The simplifying assumption of no intermediate consumption is frequently used in equilibrium asset pricing literature, including the Sharpe and Lintner's CAPM. See Bick (1990), He and Leland (1993), Grossman and Zhou (1996), Kogan et al. (2006). Since agents do not have to substitute consumption for investment, the interest rate process is not determined in equilibrium and can be specified exogenously. This allows the isolation of interest rate effects on the equilibrium dynamics. One can contrast this to production economies, in which the MPR is specified exogenously and only the interest rate is determined in equilibrium. See Dumas (1989).
} 
Another important consequence of Proposition 9.5 and Theorem 4.2 is that, under their assumptions, the following risk-return inequality ${ }^{38}$ holds:

$$
\lambda_{t}<\sigma_{t}^{S} \max _{x} \gamma^{U}(x)
$$

The reason is that MPR is driven by myopic volatility, which is smaller than the stock volatility by Theorem 8.1 and Proposition 9.5. Because of its myopic nature, equilibrium MPR "underestimates" the true stock price volatility and is unable to account for future stock price and return fluctuations. Since MPR is countercyclical, the stock is cheap in bad states (those with low $D_{t}$ ) and offers a high instantaneous return. This motivates the agents to buy more shares in those states, and the stock becomes more volatile, which, in turn, stabilizes the demand. A similar argument applies in good states. Risk-return inequality is a much weaker requirement than the risk-return equality of Merton (1973), and we can use it together with empirical data to estimate the maximal risk aversion in the economy.

\subsection{Example: Mean-Reverting Dividends and CRRA agents}

We now illustrate the results from the previous sections in our benchmark example. That is, we make the following assumption:

Assumption 9.6 The $\log$ dividend $A_{t}=\log D_{t}$ follows an autoregressive process

$$
d A_{t}=\left(a-b A_{t}\right) d t+\sigma d B_{t}
$$

interest rate $r$ is 0 , and all agents in the economy have CRRA preferences, $\gamma_{k}(x)=\gamma_{k}$.

Under Assumption 9.6, $\sigma^{D}=\sigma, c=b$. Consequently, all the formulae substantially simplify and all the dynamic properties of equilibrium are determined solely by the properties of the aggregate risk aversion $\gamma^{U}$. The following proposition summarizes our main results:

Proposition 9.7 Under Assumption 9.6,

(1) $M P R$

$$
\lambda_{t}=E_{t}^{Q}\left[\gamma^{U}\left(D_{T}\right)\right] e^{-b(T-t)} \sigma
$$

is countercyclical;

\footnotetext{
${ }^{38}$ This is indirectly related to the state price density inequality of Constantinides and Duffie (1996).
} 
(2) the stock volatility is given by

$$
\sigma_{t}^{S}=\sigma e^{-b(T-t)}+\sigma_{t}^{\text {nonmyopic }}
$$

where the nonmyopic (excess) volatility

$$
\sigma_{t}^{\text {nonmyopic }}=-\frac{e^{-b(T-t)} \sigma}{S_{t}} \operatorname{Cov}_{t}^{Q}\left(\lambda_{T}, D_{T}\right)
$$

is always positive;

(3) optimal portfolios are monotonically decreasing in risk aversion. They are given by

$$
\pi_{k t}=\frac{\lambda_{t}}{\gamma_{k} \sigma_{t}^{S}}+\pi_{k t}^{\text {hedging }}
$$

where the hedging component

$$
\pi_{k t}^{\text {hedging }}=\frac{1}{W_{k t}}\left(\gamma_{k}^{-1}-1\right) \frac{e^{-b(T-t)} \sigma}{\sigma_{t}^{S}} \operatorname{Cov}_{t}^{Q}\left(\lambda_{T}, W_{k T}\right)
$$

is positive if and only if $\gamma_{k}>1$.

We conclude this section with another interesting result related to heterogeneity in risk aversions, showing how the size of the risk-return tradeoff, the size of the ratio $\sigma_{t}^{S} / \sigma_{t}^{\text {myopic }}$ and the size of the equilibrium optimal portfolios depends on the magnitude of heterogeneity. Let

$$
\gamma \stackrel{\text { def }}{=} \min _{k} \gamma_{k}, \Gamma \stackrel{\text { def }}{=} \max _{k} \gamma_{k}
$$

Proposition 9.8 The following is true:

(1) the MPR satisfies

$$
\gamma \sigma e^{-b(T-t)} \leq \lambda_{t} \leq \Gamma \sigma e^{-b(T-t)}
$$

(2) the stock volatility satisfies

$$
\sigma e^{-b(T-t)} \leq \sigma_{t}^{S} \leq \sigma e^{-b(T-t)}(1+\Gamma-\gamma)
$$

(3) the risk-return tradeoff satisfies

$$
\frac{\gamma}{1+\Gamma-\gamma} \leq \frac{\lambda_{t}}{\sigma_{t}^{S}} \leq \Gamma
$$


(4) the optimal portfolios satisfy

$$
\begin{aligned}
& \frac{1}{\gamma_{k}} \frac{\gamma}{1+\Gamma-\gamma} \leq \pi_{k t} \leq \frac{1}{\gamma_{k}}\left(\Gamma+\left(\gamma_{k}-1\right)(\Gamma-\gamma)\right) \text { if } \gamma_{k}>1, \\
& \frac{1}{\gamma_{k}} \frac{\gamma}{1+\Gamma-\gamma} \leq \pi_{k t} \leq \frac{1}{\gamma_{k}} \Gamma \quad \text { if } \gamma_{k}<1 .
\end{aligned}
$$

These results imply that the size $\Gamma-\gamma$ of heterogeneity plays a crucial role for determining the size of excess volatility, risk-return tradeoff, and the size of equilibrium optimal portfolios. In the terminology of Dumas, Kurshev, and Uppal (2009), investors are taking advantage of heterogeneous risk attitudes in the economy, which generates excess volatility and stochastic fluctuations of the MPR.

It follows from the asymptotic results of Cvitanic and Malamud (2010b) that the bounds (25) and (26) are tight. Therefore, they are especially useful for empirical analysis because they do not depend on the parameters of the dividend process. For example, finding the empirical range

$$
\left[\min \frac{\lambda_{t}}{\sigma_{t}^{S}}, \max \frac{\lambda_{t}}{\sigma_{t}^{S}}\right]
$$

of the risk-return tradeoff allows us to gauge the size $\Gamma-\gamma$ of heterogeneity in the economy. Moreover, given the estimates for $\gamma$ and $\Gamma$ from risk-return tradeoffs, data on empirical portfolio holdings together with bounds (26) could be used to study the cross-sectional distribution of risk aversions in the economy.

\section{Conclusions}

We study nonmyopic optimal portfolios in viable markets, characterized by the fact that the state price density and the interest rate are deterministic functions of the single diffusion state variable. Our analysis is based on new representations for optimal portfolios in terms of expected values and covariances of directly observable quantities under the riskneutral measure. In these representations, macroeconomic risks are priced discounted at a specific rate, that we call the "rate of macroeconomic fluctuation". Our representations are universal because they hold in any exchange economy with an arbitrary Markovian dividend, arbitrary stochastic interest rates, and, in the equilibrium context, arbitrary rational agents maximizing utility from terminal consumption.

We derive tight conditions for the monotonicity of optimal portfolios in risk aversion and the positivity of the MPR hedge. In particular, we show that the MPR hedge consists 
of two components, the wealth hedge and the risk tolerance hedge. Those are determined respectively by the covariances of agent's future wealth and future risk tolerance with future MPR, discounted at the rate of macroeconomic fluctuation. The latter can be directly estimated from data, because the drift of the MPR is equal to the product of the MPR and its volatility plus the rate of macroeconomic fluctuation.

Our results highlight general mechanisms behind the phenomena of MPR countercyclicality, nonmyopic (excess) volatility, and nonmyopic optimal portfolios. In particular, we show that the nonmyopic volatility is determined by the interplay between the MPR and interest rate cyclicality, that the size and the sign of the interest rate hedge are determined by the interest rate volatility and the size of agent's risk aversion, whereas the size and the sign of MPR hedge are determined by the interplay between prudence and risk tolerance.

We believe our results are important for understanding single-agent (partial equilibrium) nonmyopic portfolio behavior and for understanding continuous-time equilibrium CAPM. They can be used in theory for testing various versions of CAPM and in practice for calculating optimal portfolios for long-run investors.

\section{Appendix}

\section{A Proofs: Stock Price Dynamics}

Denote by $\mathcal{D}_{t}$ the Malliavin derivative operator. ${ }^{39}$ The following is the main technical result of the paper.

\footnotetext{
${ }^{39}$ For an expedient introduction to Malliavin derivatives, see Detemple, Garcia, and Rindisbacher (2003).
} 
Proposition A.1 The drift and volatility of the stock price are given by

$$
\begin{aligned}
\mu_{t}^{S} & =r\left(D_{t}\right)+\sigma_{t}^{S}\left(E_{t}^{Q}\left[\gamma^{U}\left(D_{T}\right)\left(\mathcal{D}_{t} D_{T}\right) / D_{T}\right]\right. \\
& \left.-\int_{t}^{T} E_{t}^{Q}\left[r^{\prime}\left(D_{s}\right)\left(\mathcal{D}_{t} D_{s}\right)\right] d s\right) \\
\sigma_{t}^{S} & =\frac{1}{E_{t}\left[\xi_{T} D_{T}\right]} E_{t}\left[\left(1-\gamma_{U}\left(D_{T}\right) \xi_{T} \mathcal{D}_{t} D_{T}\right)\right] \\
& -\frac{E_{t}\left[e^{\int_{0}^{T} r\left(D_{s}\right) d s}\left(\int_{t}^{T} r^{\prime}\left(D_{s}\right)\left(\mathcal{D}_{t} D_{s}\right) d s\right) \xi_{T}\right]+E_{t}\left[e^{\int_{0}^{T} r\left(D_{s}\right) d s} \mathcal{D}_{t} \xi_{T}\right]}{E_{t}\left[e^{\int_{0}^{T} r\left(D_{s}\right) d s} \xi_{T}\right]},
\end{aligned}
$$

and the optimal portfolio of agent $k$ is given by

$$
\begin{aligned}
\pi_{k t} & =\frac{1}{\sigma_{t}^{S}} \frac{E_{t}\left[\mathcal{D}_{t} \xi_{T}\left(y_{k} \xi_{T} I_{k}^{\prime}\left(y_{k} \xi_{T}\right)+I_{k}\left(y_{k} \xi_{T}\right)\right)\right]}{E_{t}\left[\xi_{T} I_{k}\left(y_{k} \xi_{T}\right)\right]} \\
& -\frac{E_{t}\left[e^{\int_{0}^{T} r\left(D_{s}\right) d s}\left(\int_{t}^{T} r^{\prime}\left(D_{s}\right)\left(\mathcal{D}_{t} D_{s}\right) d s\right) \xi_{T}\right]+E_{t}\left[e^{\int_{0}^{T} r\left(D_{s}\right) d s} \mathcal{D}_{t} \xi_{T}\right]}{E_{t}\left[e^{\int_{0}^{T} r\left(D_{s}\right) d s} \xi_{T}\right]}
\end{aligned}
$$

where

$$
\mathcal{D}_{t} D_{T}=D_{t} \sigma^{D}\left(D_{t}\right) e^{\delta_{T}-\delta_{t}}
$$

with

$$
\begin{aligned}
\delta_{t}=\int_{0}^{t}\left[D_{s}\left(\mu^{D}\right)^{\prime}\left(D_{s}\right)-0.5\left(D_{s}\left(\sigma^{D}\right)^{\prime}\left(D_{s}\right)\right)^{2}-D_{s}\left(\sigma^{D}\right)^{\prime}\left(D_{s}\right) \sigma^{D}\left(D_{s}\right)\right] d s & \\
& +\int_{0}^{t} D_{s}\left(\sigma^{D}\right)^{\prime}\left(D_{s}\right) d B_{s}
\end{aligned}
$$

and

$$
\mathcal{D}_{t} \xi_{T}=-\frac{1}{D_{T}} \gamma^{U}\left(D_{T}\right) \xi_{T} \mathcal{D}_{t} D_{T}
$$

Proof of Proposition A.1. By definition,

$$
\xi_{t}=e^{-\int_{0}^{t} r\left(D_{s}\right) d s} M_{t}=e^{-\int_{0}^{t} r\left(D_{s}\right) d s} E_{t}\left[M_{T}\right]=E_{t}\left[e^{\int_{t}^{T} r\left(D_{s}\right) d s} \xi_{T}\right] .
$$

The price $S_{t}$ and the wealth of agent $k$ satisfy

$$
\log S_{t}=\int_{0}^{t} r\left(D_{s}\right) d s+\log E_{t}\left[\xi_{T} D_{T}\right]-\log E_{t}\left[e^{\int_{0}^{T} r\left(D_{s}\right) d s} \xi_{T}\right]
$$


and

$$
\log W_{k t}=\int_{0}^{t} r\left(D_{s}\right) d s+\log E_{t}\left[\xi_{T} I_{k}\left(y_{k} \xi_{T}\right)\right]-\log E_{t}\left[e^{\int_{0}^{T} r\left(D_{s}\right) d s} \xi_{T}\right]
$$

We get the volatility $\sigma_{t}^{S}$ as the Malliavin derivative $\mathcal{D}_{t} \log S_{t}$ and we get $\sigma_{t}^{S} \pi_{k t}$ as the Malliavin derivative $\mathcal{D}_{t} \log W_{k t}$. Thus, we have

$$
\pi_{k t}=\frac{\mathcal{D}_{t} \log W_{k t}}{\mathcal{D}_{t} \log S_{t}}
$$

We will now calculate the Malliavin derivatives. For process $D$, it is well known that the Malliavin derivative

$$
Y_{t}:=\mathcal{D}_{t} D_{u}, \quad u \geq t
$$

satisfies the linear stochastic differential equation

$$
d Y_{u}=\left(D_{u}\left(\mu^{D}\right)^{\prime}\left(D_{u}\right)+\mu^{D}\left(D_{u}\right)\right) Y_{u} d u+\left(D_{u}\left(\sigma^{D}\right)^{\prime}\left(D_{u}\right)+\sigma^{D}\left(D_{u}\right)\right) Y_{u} d B_{u}, u \geq t
$$

with

$$
Y_{t}=D_{t} \sigma^{D}\left(D_{t}\right)
$$

and (28) follows. Therefore,

$$
\mathcal{D}_{t} \xi_{T}=U^{\prime \prime}\left(D_{T}\right) \mathcal{D}_{t} D_{T} .
$$

Using the identity

$$
\mathcal{D}_{t} E_{t}[X]=E_{t}\left[\mathcal{D}_{t} X\right]
$$

we arrive at

$$
\begin{aligned}
\mathcal{D}_{t} \log W_{k t} & =\frac{1}{E_{t}\left[\xi_{T} I_{k}\left(y_{k} \xi_{T}\right)\right]} E_{t}\left[y_{k} \xi_{T} I_{k}^{\prime}\left(y_{k} \xi_{T}\right) \mathcal{D}_{t} \xi_{T}+I_{k}\left(y_{k} \xi_{T}\right) \mathcal{D}_{t} \xi_{T}\right] \\
& -\frac{E_{t}\left[e^{\int_{0}^{T} r\left(D_{s}\right) d s}\left(\int_{t}^{T} r^{\prime}\left(D_{s}\right)\left(\mathcal{D}_{t} D_{s}\right) d s\right) \xi_{T}\right]+E_{t}\left[e^{\int_{0}^{T} r\left(D_{s}\right) d s} \mathcal{D}_{t} \xi_{T}\right]}{E_{t}\left[e^{\int_{0}^{T} r\left(D_{s}\right) d s} \xi_{T}\right]}
\end{aligned}
$$

and

$$
\begin{aligned}
\mathcal{D}_{t} \log S_{t} & =\frac{1}{E_{t}\left[\xi_{T} D_{T}\right]} E_{t}\left[D_{T} \mathcal{D}_{t} \xi_{T}+\xi_{T} \mathcal{D}_{t} D_{T}\right] \\
& -\frac{E_{t}\left[e^{\int_{0}^{T} r\left(D_{s}\right) d s}\left(\int_{t}^{T} r^{\prime}\left(D_{s}\right)\left(\mathcal{D}_{t} D_{s}\right) d s\right) \xi_{T}\right]+E_{t}\left[e^{\int_{0}^{T} r\left(D_{s}\right) d s} \mathcal{D}_{t} \xi_{T}\right]}{E_{t}\left[e^{\int_{0}^{T} r\left(D_{s}\right) d s} \xi_{T}\right]} .
\end{aligned}
$$


It remains to show the expression for the drift. By the martingale property, we have

$$
\frac{d E_{t}\left[\xi_{T} D_{T}\right]}{E_{t}\left[\xi_{T} D_{T}\right]}=U_{t} d W_{t}, \frac{d E_{t}\left[e^{\int_{0}^{T} r\left(D_{s}\right) d s} \xi_{T}\right]}{E_{t}\left[e^{\int_{0}^{T} r\left(D_{s}\right) d s} \xi_{T}\right]}=V_{t} d W_{t}
$$

where, by the Clarke-Ocone formula and (31),

$$
U_{t}=\frac{\mathcal{D}_{t} E_{t}\left[\xi_{T} D_{T}\right]}{E_{t}\left[\xi_{T} D_{T}\right]}=\frac{1}{E_{t}\left[\xi_{T} D_{T}\right]} E\left[\xi_{T}\left(1-\gamma_{U}\left(D_{T}\right)\right) \mathcal{D}_{t} D_{T}\right]
$$

and

$$
\begin{aligned}
& V_{t}=\frac{\mathcal{D}_{t} E_{t}\left[e^{\int_{0}^{T} r\left(D_{s}\right) d s} \xi_{T}\right]}{E_{t}\left[e_{0}^{\int_{0}^{T} r\left(D_{s}\right) d s} \xi_{T}\right]} \\
& =-\frac{E_{t}\left[e^{\int_{0}^{T} r\left(D_{s}\right) d s}\left(\gamma^{U}\left(D_{T}\right) \xi_{T} \mathcal{D}_{t} D_{T}\right) / D_{T}\right]-E_{t}\left[e^{\int_{0}^{T} r\left(D_{s}\right) d s}\left(\int_{t}^{T} r^{\prime}\left(D_{s}\right)\left(\mathcal{D}_{t} D_{s}\right) d s\right) \xi_{T}\right]}{E_{t}\left[e^{\int_{0}^{T} r\left(D_{s}\right) d s} \xi_{T}\right]} .
\end{aligned}
$$

Applying Ito's formula, we get

$$
d \log S_{t}=r\left(D_{t}\right) d t+d \log \frac{E_{t}\left[\xi_{T} D_{T}\right]}{E_{t}\left[\xi_{T}\right]}=\frac{1}{2}\left(2 r\left(D_{t}\right)+V_{t}^{2}-U_{t}^{2}\right) d t+\left(U_{t}-V_{t}\right) d W_{t} .
$$

Therefore,

$$
\mu_{t}^{S}=r\left(D_{t}\right)+\frac{1}{2}\left(V_{t}^{2}-U_{t}^{2}+\left(U_{t}-V_{t}\right)^{2}\right)=r\left(D_{t}\right)+V_{t}\left(V_{t}-U_{t}\right)
$$

and thus, by (33),

$$
\begin{aligned}
& \mu_{t}^{S}=r\left(D_{t}\right) \\
& +\frac{E_{t}\left[e_{0}^{\int_{0}^{T} r\left(D_{s}\right) d s}\left(\gamma^{U}\left(D_{T}\right) \xi_{T} \mathcal{D}_{t} D_{T}\right) / D_{T}\right]-E_{t}\left[e^{\int_{0}^{T} r\left(D_{s}\right) d s}\left(\int_{t}^{T} r^{\prime}\left(D_{s}\right)\left(\mathcal{D}_{t} D_{s}\right) d s\right) \xi_{T}\right]}{E_{t}\left[e_{0}^{\int_{0}^{T} r\left(D_{s}\right) d s} \xi_{T}\right]} \\
& \times \mathcal{D}_{t} \log S_{t} \\
& =r\left(D_{t}\right)+\sigma_{t}^{S}\left(E_{t}^{Q}\left[\gamma^{U}\left(D_{T}\right)\left(\mathcal{D}_{t} D_{T}\right) / D_{T}\right]-\int_{t}^{T} E_{t}^{Q}\left[r^{\prime}\left(D_{s}\right)\left(\mathcal{D}_{t} D_{s}\right)\right] d s\right) .
\end{aligned}
$$

The following result allows us to rewrite the Malliavin derivative $\mathcal{D}_{t} D$ without involving stochastic integrals. It has also been proved by Detemple, Garcia, and Rindisbacher (2003) in a slightly different form, but we present a derivation here for the reader's convenience. 
Lemma A.2 We have

$$
\mathcal{D}_{t} D_{T}=D_{T} \sigma^{D}\left(D_{T}\right) e^{-\int_{t}^{T} \rho_{s} d s}
$$

Proof. By Ito's formula,

$$
\begin{aligned}
\log \left(D_{T} \sigma^{D}\left(D_{T}\right)\right) & -\log \left(D_{t} \sigma^{D}\left(D_{t}\right)\right)=\int_{t}^{T}\left(\sigma^{D}\left(D_{s}\right)+D_{s}\left(\sigma^{D}\right)^{\prime}\left(D_{s}\right)\right) d B_{s} \\
& +\int_{t}^{T}\left(\left(\sigma^{D}\left(D_{s}\right)+D_{s}\left(\sigma^{D}\right)^{\prime}\left(D_{s}\right)\right) \sigma^{D}\left(D_{s}\right)^{-1} \mu^{D}\left(D_{s}\right)\right) d s \\
& +\frac{1}{2} \int_{t}^{T}\left(\left(2\left(\sigma^{D}\right)^{\prime}\left(D_{s}\right)+D_{s}\left(\sigma^{D}\right)^{\prime \prime}\left(D_{s}\right)\right) \sigma^{D}\left(D_{s}\right)\right. \\
& \left.-\left(\sigma^{D}\left(D_{s}\right)+\left(D_{s}\left(\sigma^{D}\right)^{\prime}\left(D_{s}\right)\right)^{2}\right)\right) d s .
\end{aligned}
$$

It remains to show that

$$
\rho_{s}=c\left(D_{s}\right)
$$

where

$$
\begin{aligned}
c(x) & =-x\left(\mu^{D}\right)^{\prime}(x)+x\left(\sigma^{D}\right)^{\prime}(x) \sigma^{D}(x)^{-1} \mu^{D}(x) \\
& +\left(\sigma^{D}\right)^{\prime}(x) \sigma^{D}(x) x+0.5\left(\sigma^{D}\right)^{\prime \prime}(x) x^{2} \sigma^{D}(x) .
\end{aligned}
$$

This claim can be verified by direct calculation.

Proof of Theorem 4.2. The proof follows directly by substituting (36) into (26).

We will need the following known

Lemma A.3 For any one-dimensional diffusion, the function

$$
G(t, x)=E\left[g\left(D_{T}\right) \mid D_{t}=x\right]
$$

is monotonically increasing (decreasing) in $x$ for all $t \in[0, T]$ if and only if $g(x)$ is monotonically increasing (decreasing) in $x$. Furthermore, if both $g(x)$ and $h(x)$ are increasing (or both decreasing), then

$$
E_{t}\left[g\left(D_{T}\right)\right] E_{t}\left[h\left(D_{T}\right)\right] \leq E_{t}\left[g\left(D_{T}\right) h\left(D_{T}\right)\right]
$$

If both $g$ and $h$ are strictly increasing (or both strictly decreasing), then the inequality is also strict unless $D_{T}$ is constant. If one function is increasing and the other is decreasing, the inequality reverses.

Proof. See Herbst and Pitt (1991). 
Lemma A.4 Suppose that $F$ and $G_{1}, \cdots, G_{N}$ are monotonically increasing functions. Then for any $N \in \mathbb{N}$ and any $\left\{t_{1} \leq \cdots \leq t_{N}\right\} \subset[t, T]$,

$$
E\left[F\left(X_{T}\right) G_{1}\left(X_{t_{1}}\right) \cdots G_{N}\left(X_{t_{N}}\right) \mid X_{t}=x\right]
$$

is monotonically increasing in $x$ and

$$
E_{t}\left[F\left(X_{T}\right) G_{1}\left(X_{t_{1}}\right) \cdots G_{N}\left(X_{t_{N}}\right)\right] \geq E_{t}\left[F\left(X_{T}\right)\right] E_{t}\left[G_{1}\left(X_{t_{1}}\right) \cdots G_{N}\left(X_{t_{N}}\right)\right]
$$

Proof. The proof is by induction. For $N=1$, we have

$$
E_{t}\left[F\left(X_{T}\right) G_{t_{1}}\left(X_{t_{1}}\right)\right]=E_{t}\left[E_{t_{1}}\left[F\left(X_{T}\right)\right] G_{t_{1}}\left(X_{t_{1}}\right)\right]
$$

By Lemma A.3, the function inside the expectation is increasing in $X_{t_{1}}$ and another application of Lemma A.3 provides monotonicity of $E_{t}\left[F\left(X_{T}\right) G_{t_{1}}\left(X_{t_{1}}\right)\right]$. Now, by Lemma A.3,

$$
\begin{aligned}
E_{t}\left[E_{t_{1}}\left[F\left(X_{T}\right)\right] G_{1}\left(X_{t_{1}}\right)\right] & \geq E_{t}\left[E_{t_{1}}\left[F\left(X_{T}\right)\right]\right] E_{t}\left[G_{t_{1}}\left(X_{t_{1}}\right)\right] \\
& =E_{t}\left[F\left(X_{T}\right)\right] E_{t}\left[G_{t_{1}}\left(X_{t_{1}}\right)\right],
\end{aligned}
$$

and we are done. Suppose now that the claim has been proved for $N$. Then

$$
\begin{aligned}
E_{t}\left[F\left(X_{T}\right) G_{1}\left(X_{t_{1}}\right)\right. & \left.\cdots G_{N}\left(X_{t_{N}}\right)\right] \\
& =E_{t}\left[G_{1}\left(X_{t_{1}}\right) E_{t_{1}}\left[F\left(X_{T}\right) G_{2}\left(X_{t_{2}}\right) \cdots G_{N}\left(X_{t_{N}}\right)\right]\right] \\
& \geq E_{t}\left[E_{t_{1}}\left[F\left(X_{T}\right)\right] E_{t_{1}}\left[G_{1}\left(X_{t_{1}}\right) G_{2}\left(X_{t_{2}}\right) \cdots G_{N}\left(X_{t_{N}}\right)\right]\right]
\end{aligned}
$$

and the claim follows from Lemma A.3 and the induction hypothesis.

Lemma A.5 Suppose that $f$ and $g$ are both increasing (decreasing). Then the following is true:

(1) the function ${ }^{40}$

$$
E\left[f\left(D_{T}\right) e^{\int_{t}^{T} g\left(D_{s}\right) d s} \mid D_{t}=x\right]
$$

is also monotonically increasing (decreasing);

(2) we have

$$
\operatorname{Cov}_{t}\left(h\left(D_{T}\right), f\left(D_{T}\right) e^{\int_{t}^{T} g\left(D_{s}\right) d s}\right) \geq 0
$$

if $h$ has the same direction of monotonicity as $f$ and $g$, and the inequality reverses if $h$ has the opposite direction of monotonicity.

\footnotetext{
${ }^{40}$ Item (1) of Lemma A.5 is contained in Mele (2007).
} 
Proof of Lemma A.5. The claim follows from Lemma A.4, approximating the integral $\int_{t}^{T} g\left(D_{s}\right) d s$ by discrete integral sums.

Proof of Theorem 8.1. The proof follows directly from Proposition A.1 and (36). The fact that $\sigma_{t}^{S}>0$ when $r^{\prime}$ is sufficiently small follows from (2), Lemma A.5 and

$$
\sigma_{t}^{S}=\frac{\partial}{\partial D_{t}} S\left(t, D_{t}\right) \sigma^{D}\left(D_{t}\right)
$$

\section{B Proofs: Optimal Portfolios}

We start with the following auxiliary result:

Proposition B.1 The optimal portfolio $\pi_{k t}$ is given by

$$
\sigma_{t}^{S} \pi_{k t}=\frac{E_{t}^{Q}\left[\lambda(t, T) e^{-\int_{t}^{T} r\left(D_{s}\right) d s} W_{k T}\left(\gamma_{k}^{-1}\left(W_{k T}\right)-1\right)\right]}{W_{k t}}+\lambda_{t} .
$$

Proof of Proposition B.1. The claim follows directly from Proposition A.1 and (36).

Proof of Proposition 5.1. Let $t=0$ for simplicity. By definition, the value function is

$$
U_{k}(x)=E\left[u_{k}\left(I_{k}\left(\xi_{T} y_{k}\right)\right)\right]
$$

where $y_{k}=y_{k}(x)$ solves

$$
x=E\left[\xi_{T} I_{k}\left(\xi_{T} y_{k}\right)\right]
$$

Differentiating this identity, we get

$$
y_{k}^{\prime}(x)=E\left[\xi_{T}^{2} I_{k}^{\prime}\left(\xi_{T} y_{k}\right)\right]^{-1}
$$

and therefore

$$
U_{k}^{\prime}(x)=E\left[u_{k}^{\prime}\left(I_{k}\left(\xi_{T} y_{k}\right)\right) I_{k}^{\prime}\left(\xi_{T} y_{k}\right) \xi_{T}\right] y_{k}^{\prime}(x)=E\left[\xi_{T} y_{k} I_{k}^{\prime}\left(\xi_{T} y_{k}\right) \xi_{T}\right] y_{k}^{\prime}(x)=y_{k}(x)
$$

Consequently,

$$
U_{k}^{\prime \prime}(x)=y_{k}^{\prime}(x)=\frac{1}{E\left[\xi_{T}^{2} I_{k}^{\prime}\left(\xi_{T} y_{k}\right)\right]}
$$

and

$$
\gamma_{k 0}(x)=-\frac{x}{y_{k} E\left[\xi_{T}^{2} I_{k}^{\prime}\left(\xi_{T} y_{k}\right)\right]}=-\frac{E\left[\xi_{T} I_{k}\left(\xi_{T} y_{k}\right)\right]}{E\left[\xi_{T}^{2} y_{k} I_{k}^{\prime}\left(\xi_{T} y_{k}\right)\right]} .
$$


Differentiating the identity

$$
u_{k}^{\prime}\left(I_{k}(x)\right)=x
$$

we get

$$
I_{k}^{\prime}(x)=\left(u_{k}^{\prime \prime}(x)\right)^{-1}
$$

and therefore

$$
y_{k} \xi_{T} I_{k}^{\prime}\left(y_{k} \xi_{T}\right)=-\gamma_{k T}^{-1} W_{k T}
$$

Proof of Theorem 5.2. By Propositions B.1 and 5.1,

$$
\begin{aligned}
\sigma_{t}^{S} \pi_{k t}^{\text {hedging }} & =\sigma_{t}^{S}\left(\pi_{k t}-\pi_{k t}^{\text {myopic }}\right) \\
& =\frac{E_{t}^{Q}\left[\gamma^{U}\left(D_{T}\right) \sigma^{D}(t, T) e^{-\int_{t}^{T} r_{s} d s} W_{k T}\left(\gamma_{k T}^{-1}-1\right)\right]}{E_{t}^{Q}\left[e^{-\int_{t}^{T} r_{s} d s} W_{k T}\right]}+\lambda_{t} \\
& -\lambda_{t} \frac{E_{t}^{Q}\left[e^{-\int_{t}^{T} r_{s} d s} \gamma_{k T}^{-1} W_{k T}\right]}{E_{t}^{Q}\left[e^{-\int_{t}^{T} r_{s} d s} W_{k T}\right]} \\
& =\frac{E_{t}^{Q}\left[\gamma^{U}\left(D_{T}\right) \sigma^{D}(t, T) e^{-\int_{t}^{T} r_{s} d s} W_{k T}\left(\gamma_{k T}^{-1}-1\right)\right]}{E_{t}^{Q}\left[e^{-\int_{t}^{T} r_{s} d s} W_{k T}\right]} \\
& -\lambda_{t} \frac{E_{t}^{Q}\left[e^{-\int_{t}^{T} r_{s} d s}\left(\gamma_{k T}^{-1}-1\right) W_{k T}\right]}{E_{t}^{Q}\left[e^{-\int_{t}^{T} r_{s} d s} W_{k T}\right]} \\
& =\frac{1}{E_{t}^{Q}\left[W_{k T}\right]}\left(E_{t}^{Q}\left[\gamma^{U}\left(D_{T}\right) \sigma^{D}(t, T) e^{-\int_{t}^{T} r_{s} d s} W_{k T}\left(\gamma_{k T}^{-1}-1\right)\right]\right. \\
& \left.-E_{t}^{Q}\left[\gamma^{U}\left(D_{T}\right) \sigma^{D}(t, T)\right] E_{t}^{Q}\left[\left(\gamma_{k T}^{-1}-1\right) e^{-\int_{t}^{T} r_{s} d s} W_{k T}\right]\right) \\
& +\frac{E_{t}^{Q}\left[e^{-\int_{t}^{T} r_{s} d s}\left(\gamma_{k T}^{-1}-1\right) W_{k T}\right]}{E_{t}^{Q}\left[e^{-\int_{t}^{T} r_{s} d s} W_{k T}\right]}\left[\int_{t}^{T} \sigma^{r}(t, \tau) d \tau\right]
\end{aligned}
$$

Q.E.D.

Lemma B.2 Suppose $\gamma_{i}(x)<a$ for some $a>0$. Then there exists a smooth oneparameter family of utilities $u(x, \beta)$ such that $u(x, 0)=u_{i}(x), u(x, 1)=x^{1-a} /(1-a)$ and the function $I(x, \beta) \stackrel{\text { def }}{=}\left(u^{\prime}(x, \beta)\right)^{-1}$ is such that

$$
\frac{-I_{x}(x, \beta) x}{I(x, \beta)}
$$

is monotonically decreasing in $\beta$. 
Proof. A direct calculation shows that $I_{i}(x)=\left(u_{i}^{\prime}(x)\right)^{-1}$ satisfies

$$
-\frac{I_{i}^{\prime}(x) x}{I_{i}(x)}=-\frac{u_{i}^{\prime}\left(I_{i}(x)\right)}{u_{i}^{\prime \prime}\left(I_{i}(x)\right) I_{i}(x)}=1 / \gamma_{i}\left(I_{i}(x)\right) .
$$

Defining

$$
I(x, \beta)=\exp \left(-\int_{1}^{x} 1 /\left((1-\beta) \gamma_{i}\left(I_{i}(y)\right)+\beta a\right) y^{-1} d y\right)
$$

we get

$$
\frac{-I_{x}(x, \beta) x}{I(x, \beta)}=1 /\left((1-\beta) \gamma_{i}\left(I_{i}(x)\right)+\beta a\right)
$$

which clearly satisfies the required conditions, and

$$
u^{\prime}(x, \beta)=(I(x, \beta))^{-1}
$$

is the required marginal utility family.

Proof of Theorem 6.3. Suppose that $\sup _{x} \gamma_{i}(x) \leq \inf _{x} \gamma_{j}(x)$ and let $a=$ $\sup _{x} \gamma_{i}(x)$. Then it suffices to show that the optimal portfolio holding of agent $i$ is smaller than that of an agent with constant relative risk aversion $a$ and the portfolio holdings of agent $j$ are larger than that of an agent with constant relative risk aversion $a$. We only prove the first claim, as the second claim is analogous.

Recall that $W_{i t}=I_{i}\left(y_{i} U^{\prime}\left(D_{T}\right)\right)$ where $y_{i}$ is the Lagrange multiplier for agent $i$ and therefore, by (43),

$$
\left(1-\gamma_{i}^{-1}\left(W_{i T}\right)\right) W_{i T}=I\left(y_{i} U^{\prime}\left(D_{T}\right), \beta\right)+I_{x}\left(y_{i} U^{\prime}\left(D_{T}\right), \beta\right) y_{i} U^{\prime}\left(D_{T}\right)
$$

Consider the family $I(x, \beta)$ of Lemma B.2 and define the function

$$
\phi(\beta)=\frac{E_{t}\left[\sigma\left(D_{T}\right) \gamma^{U}\left(D_{T}\right) U^{\prime}\left(D_{T}\right)\left(I\left(y_{i} U^{\prime}\left(D_{T}\right), \beta\right)+I_{x}\left(y_{i} U^{\prime}\left(D_{T}\right), \beta\right) y_{i} U^{\prime}\left(D_{T}\right)\right)\right]}{E_{t}\left[U^{\prime}\left(D_{T}\right) I\left(y_{i} U^{\prime}\left(D_{T}\right), \beta\right)\right]} .
$$

Then by Proposition B.1, we have

$$
\begin{aligned}
\phi(0) & =\frac{E_{t}\left[\sigma\left(D_{T}\right) \gamma^{U}\left(D_{T}\right) U^{\prime}\left(D_{T}\right)\left(I_{i}\left(y_{i} U^{\prime}\left(D_{T}\right)\right)+I_{i}^{\prime}\left(y_{i} U^{\prime}\left(D_{T}\right)\right) y_{i} U^{\prime}\left(D_{T}\right)\right)\right]}{E_{t}\left[U^{\prime}\left(D_{T}\right) I_{i}\left(y_{i} U^{\prime}\left(D_{T}\right)\right)\right]} \\
& =e^{\rho(T-t)}\left(-\sigma_{t}^{S} \pi_{i t}+\lambda_{t}\right) .
\end{aligned}
$$

Furthermore, a direct calculation shows that

$$
\phi(1)=\frac{\left(1-a^{-1}\right) E_{t}\left[\sigma\left(D_{T}\right) \gamma^{U}\left(D_{T}\right)\left(\left(U^{\prime}\left(D_{T}\right)\right)^{1-a^{-1}}\right)\right]}{E_{t}\left[U^{\prime}\left(D_{T}\right)\left(U^{\prime}\left(D_{T}\right)\right)^{1-a^{-1}}\right]}
$$


is independent of $y_{i}$. Therefore, by Proposition B.1,

$$
e^{\rho(T-t)}\left(\sigma_{t}^{S}\right)^{-1}\left(-\phi(1)+\lambda_{t}\right)
$$

is the optimal portfolio holdings of an agent with risk aversion $a$. Here, the rate of macroeconomic fluctuation $\rho_{t}=\rho$ is constant by assumption. Thus, it suffices to show that $\phi(\beta)$ is monotonically increasing.

Differentiating $\phi(\beta)$ with respect to $\beta$, we get

$$
\begin{aligned}
& \left(E_{t}\left[U^{\prime}\left(D_{T}\right) I\left(y U^{\prime}\left(D_{T}\right), \beta\right)\right]\right)^{2} \times \phi^{\prime}(\beta)= \\
& =E_{t}\left[\sigma\left(D_{T}\right) \gamma^{U}\left(D_{T}\right) U^{\prime}\left(D_{T}\right)\left(I_{\beta}\left(y_{i} U^{\prime}\left(D_{T}\right), \beta\right)+I_{x \beta}\left(y_{i} U^{\prime}\left(D_{T}\right), \beta\right) y_{i} U^{\prime}\left(D_{T}\right)\right)\right] \times \\
& E_{t}\left[U^{\prime}\left(D_{T}\right) I\left(y_{i} U^{\prime}\left(D_{T}\right), \beta\right)\right] \\
& -E_{t}\left[\sigma\left(D_{T}\right) \gamma^{U}\left(D_{T}\right) U^{\prime}\left(D_{T}\right)\left(I\left(y_{i} U^{\prime}\left(D_{T}\right), \beta\right)+I_{x}\left(y_{i} U^{\prime}\left(D_{T}\right), \beta\right) y_{i} U^{\prime}\left(D_{T}\right)\right)\right] \times \\
& E_{t}\left[U^{\prime}\left(D_{T}\right) I_{\beta}\left(y_{i} U^{\prime}\left(D_{T}\right), \beta\right)\right]
\end{aligned}
$$

Now, by Corollary 3.2, the assumption that $\rho_{t}$ is constant implies that $D_{T}=f\left(A_{T}\right)$ where $A_{T}$ has a Gaussian distribution. Modifying the function $f$ if necessary, we may assume without loss of generality that $A_{T} \sim N(0,1)$. Then expression (46), multiplied by $2 \pi$, is given by

$$
\begin{aligned}
& \int_{\mathbb{R}} e^{-x^{2} / 2} f^{\prime}(x)\left(-U^{\prime \prime}(f(x))\right)\left(I_{\beta}\left(y_{i} U^{\prime}(f(x)), \beta\right)+I_{x \beta}\left(y_{i} U^{\prime}(f(x)), \beta\right) y_{i} U^{\prime}(f(x))\right) d x \times \\
& \left.\int_{\mathbb{R}} e^{-x^{2} / 2} U^{\prime}(f(x)) I\left(y_{i} U^{\prime}(f(x)), \beta\right)\right) d x \\
& -\int_{\mathbb{R}} e^{-x^{2} / 2} f^{\prime}(x)\left(-U^{\prime \prime}(f(x))\right)\left(I\left(y_{i} U^{\prime}(f(x)), \beta\right)+I_{x}\left(y_{i} U^{\prime}(f(x)), \beta\right) y_{i} U^{\prime}(f(x))\right) d x \times \\
& \int_{\mathbb{R}} e^{-x^{2} / 2} U^{\prime}(f(x)) I_{\beta}\left(y_{i} U^{\prime}(f(x)), \beta\right) d x
\end{aligned}
$$

Here, we often suppress the dependence on $\beta$. Now, we have

$$
I_{\beta}(x, \beta)+I_{x \beta}(x, \beta) x d x=\frac{d}{d x}\left(I_{\beta}(x, \beta) x\right)
$$


and therefore, integrating by parts, we get

$$
\begin{aligned}
& \int_{-\infty}^{+\infty} e^{-x^{2} / 2} f^{\prime}(x)\left(-U^{\prime \prime}(f(x))\right)\left(I_{\beta}\left(y_{i} U^{\prime}(f(x)), \beta\right)+I_{x \beta}\left(y_{i} U^{\prime}(f(x)), \beta\right) y_{i} U^{\prime}(f(x))\right) d x \\
& =-\int_{-\infty}^{+\infty} e^{-x^{2} / 2} d\left(I_{\beta}\left(y_{i} U^{\prime}(f(x)), \beta\right) y_{i} U^{\prime}(f(x))\right) \\
& =-\int_{-\infty}^{+\infty} x e^{-x^{2} / 2} I_{\beta}\left(y_{i} U^{\prime}(f(x)), \beta\right) y_{i} U^{\prime}(f(x)) d x
\end{aligned}
$$

where the minus sign appears because $U^{\prime}$ is monotonically decreasing. Similarly,

$$
\begin{aligned}
& \int_{-\infty}^{+\infty} e^{-x^{2} / 2} f^{\prime}(x)\left(-U^{\prime \prime}(f(x))\right)\left(I\left(y_{i} U^{\prime}(f(x)), \beta\right)+I_{x}\left(y_{i} U^{\prime}(f(x)), \beta\right) y_{i} U^{\prime}(f(x))\right) d x \\
& =-\int_{-\infty}^{+\infty} e^{-x^{2} / 2} d\left(I\left(y_{i} U^{\prime}(f(x)), \beta\right) y_{i} U^{\prime}(f(x))\right) \\
& =-\int_{-\infty}^{+\infty} x e^{-x^{2} / 2} I\left(y_{i} U^{\prime}(f(x)), \beta\right) y_{i} U^{\prime}(f(x)) d x,
\end{aligned}
$$

Therefore, the expression in (47) is equal to

$$
\begin{aligned}
& -\int_{\mathbb{R}} x e^{-x^{2} / 2} I_{\beta}\left(y_{i} U^{\prime}(f(x)), \beta\right) y_{i} U^{\prime}(f(x)) d x \int_{\mathbb{R}} e^{-x^{2} / 2} U^{\prime}(f(x)) I\left(y_{i} U^{\prime}(f(x)), \beta\right) d x \\
& +\int_{\mathbb{R}} x e^{-x^{2} / 2} I\left(y_{i} U^{\prime}(f(x)), \beta\right) y_{i} U^{\prime}(f(x)) d x \int_{\mathbb{R}} e^{-x^{2} / 2} U^{\prime}(f(x)) I_{\beta}\left(y_{i} U^{\prime}(f(x)), \beta\right) d x .
\end{aligned}
$$

By Lemma B.2,

$$
0>-\frac{\partial}{\partial \beta} \frac{I_{x}(x, \beta)}{I(x, \beta)}=-\frac{I_{x \beta} I-I_{x} I_{\beta}}{I^{2}} x
$$

that is

$$
I_{x \beta} I>I_{x} I_{\beta} \Leftrightarrow \frac{\partial}{\partial x} \frac{I_{\beta}}{I}>0 .
$$

Consequently, the function

$$
\psi(x)=\frac{I_{\beta}\left(y_{i} U^{\prime}(f(x)), \beta\right)}{I\left(y_{i} U^{\prime}(f(x)), \beta\right)}
$$

is monotonically decreasing in $x$.

Introduce a new probability measure

$$
d \tilde{\mu}=\frac{e^{-x^{2} / 2} U^{\prime}(f(x)) I\left(y_{i} U^{\prime}(f(x)), \beta\right) d x}{\int_{\mathbb{R}} e^{-x^{2} / 2} U^{\prime}(f(x)) I\left(y_{i} U^{\prime}(f(x)), \beta\right) d x} .
$$


Then we can rewrite expression (50) in the form

$$
\left(\int_{\mathbb{R}} e^{-x^{2} / 2} U^{\prime}(f(x)) I\left(y_{i} U^{\prime}(f(x)), \beta\right) d x\right)^{2}\left(-E^{\tilde{\mu}}\left[A_{T} \psi\left(A_{T}\right)\right]+E^{\tilde{\mu}}\left[A_{T}\right] E^{\tilde{\mu}}\left[\psi\left(A_{T}\right)\right]\right)
$$

which is nonnegative by Lemma A.3.

Proof of Theorem 6.5. By (41), we need to show that

$$
\begin{gathered}
\frac{E_{t}^{Q}\left[\gamma^{U}\left(D_{T}\right) \sigma^{D}\left(D_{T}\right) e^{-\int_{t}^{T}\left(\rho_{s}+r_{s}\right) d s} W_{k T}\left(1-\gamma_{k}^{-1}\left(W_{k T}\right)\right)\right]}{E_{t}^{Q}\left[e^{-\int_{t}^{T} r_{s} d s} W_{k T}\right]} \\
\geq \frac{E_{t}^{Q}\left[\gamma^{U}\left(D_{T}\right) \sigma^{D}\left(D_{T}\right) e^{-\int_{t}^{T}\left(\rho_{s}+r_{s}\right) d s} W_{j T}\left(1-\gamma_{j}^{-1}\left(W_{j T}\right)\right)\right]}{E_{t}^{Q}\left[e^{-\int_{t}^{T} r_{s} d s} W_{j T}\right]} .
\end{gathered}
$$

We only prove case (1). Case (2) is analogous. Since, by assumption,

$$
\inf \left(1-\gamma_{k T}^{-1}\right) \geq \sup \left(1-\gamma_{j T}^{-1}\right)
$$

it suffices to show that

$$
\frac{E_{t}^{Q}\left[\gamma^{U}\left(D_{T}\right) \sigma^{D}\left(D_{T}\right) e^{-\int_{t}^{T}\left(\rho_{s}+r_{s}\right) d s} W_{k T}\right]}{E_{t}^{Q}\left[e^{-\int_{t}^{T} r_{s} d s} W_{k T}\right]} \geq \frac{E_{t}^{Q}\left[\gamma^{U}\left(D_{T}\right) \sigma^{D}\left(D_{T}\right) e^{-\int_{t}^{T}\left(\rho_{s}+r_{s}\right) d s} W_{j T}\right]}{E_{t}^{Q}\left[e^{-\int_{t}^{T} r_{s} d s} W_{j T}\right]} .
$$

Introduce a new probability measure

$$
d Q^{k}=\frac{e^{-\int_{t}^{T} r_{s} d s} W_{k T}}{E^{Q}\left[e^{-\int_{t}^{T} r_{s} d s} W_{k T}\right]} d Q
$$

and let

$$
f(x)=\frac{I_{j}\left(y_{j} x\right)}{I_{k}\left(y_{k} x\right)} .
$$

and $z_{i}=I_{i}\left(\lambda_{i} x\right), i \in\{j, k\}$. Then

$$
\begin{aligned}
f^{\prime}(x) & =\frac{I_{j}\left(y_{j} x\right)}{x I_{k}\left(y_{k} x\right)}\left(y_{j} x \frac{I_{j}^{\prime}\left(y_{j} x\right)}{I_{j}\left(y_{j} x\right)}-y_{k} x \frac{I_{k}^{\prime}\left(y_{j} x\right)}{I_{k}\left(y_{k} x\right)}\right) \\
& =\frac{I_{j}\left(y_{j} x\right)}{x I_{k}\left(y_{k} x\right)}\left(\frac{u_{j}^{\prime}\left(z_{1}\right)}{z_{1} u_{j}^{\prime \prime}\left(z_{1}\right)}-\frac{u_{k}^{\prime}\left(z_{2}\right)}{z_{2} u_{k}^{\prime \prime}\left(z_{2}\right)}\right) \\
& =\frac{z_{1}}{x z_{2}}\left(\gamma_{k}^{-1}\left(z_{2}\right)-\gamma_{j}^{-1}\left(z_{1}\right)\right) \leq 0 .
\end{aligned}
$$


That is, $f$ is decreasing. Therefore, $f\left(U^{\prime}\left(D_{T}\right)\right)$ is increasing and, by Lemma A.5,

$$
\begin{aligned}
& \frac{E_{t}^{Q}\left[\gamma^{U}\left(D_{T}\right) \sigma^{D}\left(D_{T}\right) e^{-\int_{t}^{T}\left(\rho_{s}+r_{s}\right) d s} W_{j T}\right]}{E_{t}^{Q}\left[e^{-\int_{t}^{T} r_{s} d s} W_{j T}\right]} \\
& =\frac{E_{t}^{Q_{k}}\left[\gamma^{U}\left(D_{T}\right) \sigma^{D}\left(D_{T}\right) e^{-\int_{t}^{T} \rho_{s} d s} f\left(U^{\prime}\left(D_{T}\right)\right)\right]}{E_{t}^{Q_{k}}\left[f\left(U^{\prime}\left(D_{T}\right)\right)\right]} \\
& \leq E_{t}^{Q_{k}}\left[\gamma^{U}\left(D_{T}\right) \sigma^{D}\left(D_{T}\right) e^{\left.-\int_{t}^{T} \rho_{s} d s\right]}=\frac{E_{t}^{Q}\left[\gamma^{U}\left(D_{T}\right) \sigma^{D}\left(D_{T}\right) e^{-\int_{t}^{T}\left(\rho_{s}+r_{s}\right) d s} W_{k T}\right]}{E_{t}^{Q}\left[e^{-\int_{t}^{T} r_{s} d s} W_{k T}\right]} .\right.
\end{aligned}
$$

Proofs of Proposition 9.4, Proposition 9.5 and Theorem 7.1. Propositions 9.4 and 9.5 follow directly from Lemma A.5. Theorem 7.1 follows from Theorem 5.2 and Lemma A.5 since

$$
f(x)=x-R_{k}(x)
$$

is increasing if and only if

$$
f^{\prime}(x)=1+\frac{\left(u_{k}^{\prime \prime}\right)^{2}-u_{k}^{\prime}(x) u_{k}^{\prime \prime \prime}(x)}{\left(u_{k}^{\prime \prime}(x)\right)^{2}}=\frac{1}{\left(u_{k}^{\prime \prime}(x)\right)^{2}}\left(2-P_{k}(x) R_{k}(x)\right) \geq 0
$$

and is decreasing otherwise.

Proof of Proposition 9.8. Item (1) follows from $\gamma^{U} \in[\gamma, \Gamma]$. Items (2) and (3) follow from

$$
\begin{aligned}
0 & \leq-\left(E_{t}^{Q}\left[D_{T}\right]\right)^{-1} \operatorname{Cov}_{t}^{Q}\left(\gamma^{U}\left(D_{T}\right), D_{T}\right) \\
& =-\frac{E_{t}^{Q}\left[\gamma^{U}\left(D_{T}\right) D_{T}\right]}{E_{t}^{Q}\left[D_{T}\right]}+E_{t}^{Q}\left[\gamma^{U}\left(D_{T}\right)\right] \leq-\gamma+\Gamma
\end{aligned}
$$

Similarly,

$$
\begin{aligned}
0 & \leq-\left(E_{t}^{Q}\left[W_{k T}\right]\right)^{-1} \operatorname{Cov}_{t}^{Q}\left(\gamma^{U}\left(D_{T}\right), W_{k T}\right) \\
& =-\frac{E_{t}^{Q}\left[\gamma^{U}\left(D_{T}\right) W_{k T}\right]}{E_{t}^{Q}\left[W_{k T}\right]}+E_{t}^{Q}\left[\gamma^{U}\left(D_{T}\right)\right] \leq-\gamma+\Gamma
\end{aligned}
$$

and items (2)-(3) immediately yield the required inequality for $\gamma_{k}>1$. The case $\gamma_{k}<1$ follows from the directly verifiable identity

$$
\pi_{k t}=\frac{\sigma e^{-b(T-t)}}{\sigma_{t}^{S}}\left(\gamma_{k}^{-1} \frac{E_{t}^{Q}\left[\gamma^{U}\left(D_{T}\right) W_{k T}\right]}{E_{t}^{Q}\left[W_{k T}\right]}-\operatorname{Cov}_{t}^{Q}\left(\gamma^{U}\left(D_{T}\right), W_{k T}\right)\right)
$$


Example B.3 Suppose that the stock price $S_{t}$ takes value 1 at time 0 , values $(u, d)=$ $(1.5,0.5)$ with probabilities 0.5 at time 1 , and the interest rate is 0 . In the second period, conditioned on $u$ (resp. d), $S_{2} / S_{1}=\left(u_{1}, d_{1}\right)$ (resp. $\left(u_{2}, d_{2}\right)$ ). Let us denote by $\pi_{0 \gamma}$ the optimal stock weight at time 0 of a CRRA investor with risk aversion $\gamma$. Then a direct calculation shows that

$$
\pi_{0 \gamma}=-2+\frac{4}{1+\frac{m_{2}^{1-b}+\left(2-m_{2}\right)^{1-b}}{m_{1}^{1-b}+\left(2-m_{1}\right)^{1-b}}}
$$

where $m_{1}=\frac{2\left(1-d_{1}\right)}{u_{1}-d_{1}}, m_{2}=\frac{2\left(1-d_{2}\right)}{u_{2}-d_{2}}$ and $b=\gamma^{-1}$. Clearly, by choosing different $\left(u_{1}, d_{1}\right)$ and $\left(u_{2}, d_{2}\right)$ we can achieve any desirable monotonicity behavior in $\gamma$.

\section{References}

[1] Arrow, Kenneth J. (1965): Aspects of the Theory of Risk-Bearing. Helsinki: Yrjö Hahnsson Foundation.

[2] Aura, Saku, Peter Diamond and John Geanakoplos (2002): "Savings and Portfolio Choice in a Two-Period Two-Asset Model," American Economic Review, 92(4), 1185-1191.

[3] Barberis, Nicholas (2000): "Investing for the long run when returns are predictable," Journal of Finance, 55, 225-264.

[4] Basak, Suleyman (2005): "Asset Pricing with Heterogeneous Beliefs," Journal of Banking and Finance, 29, 2849-2881.

[5] Basak, Suleyman and Domenico Cuoco (1998): "An Equilibrium Model with Restricted Stock Market Participation," Review of Financial Studies, 11, 309341.

[6] Bhamra, Harjoat S. and Raman Uppal (2009): "The Effect of Introducing a NonRedundant Derivative on the Volatility of Stock-Market Returns When Agents Differ in Risk Aversion," Review of Financial Studies, 22, 2303-2330.

[7] Benninga, Simon and Joram Mayshar (2000): "Heterogeneity and Option Pricing," Review of Derivatives Research, 4(1), 7-27.

[8] Berrada, Tony, Julien Hugonnier and Marcel Rindisbacher (2007): "Heterogenous Preferences and Equilibrium Trading Volume," Journal of Financial Economics, 83, 719-750. 
[9] Biais, Bruno, Bossaerts, Peter and Chester Spatt (2009): "Equilibrium Asset Pricing And Portfolio Choice Under Asymmetric Information," forthcoming in The Review of Financial Studies.

[10] Bick, Avi (1990): "On Viable Diffusion Price Processes of the Market Portfolio," Journal of Finance, 15, 673-689.

[11] Brunnermeier, Markus and Stefan Nagel (2008): "Do Wealth Fluctuations Generate Time-Varying Risk Aversion? Micro-Evidence on Individuals' Asset Allocation," American Economic Review, 98(3), 713-736.

[12] Campbell, John Y. and John Cochrane (1999): "By Force of Habit: A Consumption-Based Explanation of Aggregate Stock Market Behavior," Journal of Political Economy, 107, 205-251.

[13] Campbell, John and Louis Viciera (1999): "Consumption and Portfolio Decisions when Expected Returns are Time Varying," Quarterly Journal of Economics, 114, 433-495.

[14] Constantinides, George and Darrell Duffie (1996): "Asset Pricing with Heterogeneous Consumers," Journal of Political Economy, 104(2), 219-240.

[15] Kaniel, Ron and Domenico Cuoco (2010): "Equilibrium Prices in the presence of Delegated Portfolio Management," forthcoming in The Journal of Financial Economics.

[16] Cvitanić, Jaksa and Semyon Malamud (2010a): "Relative Extinction of Heterogeneous Agents," The B. E. Journal of Theoretical Economics, 10(1).

[17] Cvitanić, Jaksa and Semyon Malamud (2010b): "Price Impact and Portfolio Impact," forthcoming in The Journal of Financial Economics

[18] Cvitanić, Jaksa, Elýes Jouini, Semyon Malamud and Clotilde Napp (2009): "Financial Markets Equilibrium with Heterogeneous Agents," working paper.

[19] Dana, Rose-Anne (1995): “An Extension of Milleron, Mitjushin and Polterovich's Result," Journal of Mathematical Economics, 24(3), 259-269.

[20] Dana, Rose-Anne (2001): "Risk Aversion and Uniqueness of Equilibrium. An Application to Financial Markets," Review of Economic Design, 6, 155-173 (special issue in the honor of R. Radner, edited by Ischiishi., T. et Marshak, T.). 
[21] Detemple, Jérôme B., René Garcia and Marcel Rindisbacher (2003): "A Monte Carlo Method for Optimal Portfolios," The Journal of Finance, 58, 401-446.

[22] Detemple, Jérôme B. and Marcel Rindisbacher (2005): "Closed Form Solutions for Optimal Portfolio Selection with Stochastic Interest Rate and Investment Constraints," Mathematical Finance, 15(4), 539-568.

[23] Detemple, Jérôme B. and Marcel Rindisbacher (2009): "Dynamic Asset Allocation: Portfolio Decomposition Formula and Applications," Review of Financial Studies, 23(1), 25-100.

[24] Detemple, Jérôme B. and Fernando Zapatero (1991): "Asset Prices in an Exchange Economy with Habit Formation," Econometrica, 59, 1633-1657.

[25] Duffie, Darrell and Chi-Fu Huang (1986): "Implementing Arrow-Debreu Equilibria by Continuous Trading of Few Long-Lived Securities," Econometrica, 53(6), 1337-1356.

[26] Duffie, Darrell (2001): Dynamic Asset Pricing Theory, 3rd ed. Princeton, New Jersey: Princeton University Press.

[27] Dumas, Bernard (1989): "Two-Person Dynamic Equilibrium in the Capital Market," Review of Financial Studies, 2, 157-188.

[28] Dumas, Bernard, Jeff Fleming and Robert E. Whaley (1998): "Implied Volatility Functions: Empirical Tests," Journal of Finance, 53(6), 2059-2106.

[29] Dumas, Bernard, Alexander Kurshev and Raman Uppal (2008): "Equilibrium Portfolio Strategies in the Presence of Sentiment Risk and Excess Volatility," Journal of Finance, 64(2), 579-629.

[30] Fama, Eugene F. and Kenneth R. French (1989): "Business Conditions and Expected Returns on Stocks and Bonds," Journal of Financial Economics, 25, 23-49.

[31] Ferson, William E. and Campbell R. Harvey (1991): "The Variation of Economic Risk Premiums," Journal of Political Economy, 99, 385-415.

[32] French, Mark W. and Daniel E. Sichel (1993): "Cyclical Patterns in the Variance of Economic Activity," Journal of Business and Economic Statistics, 11(1), 113119. 
[33] Gennotte, Gerard and Terry A. Marsh (1993): "Variations in Economic Uncertainty and Risk Premiums on Capital Assets," European Economic Review, 37(5), 1021-1041.

[34] Glosten, Lawrence R., Ravi Jagannathan, and David E. Runkle (1993): "On the Relation between the Expected Value and the Volatility of the Nominal Excess Return on Stocks," Journal of Finance, 48(5), 1779-1801.

[35] Gollier, Christian and John W. Pratt (1996): "Risk Vulnerability and the Tempering Effect of Background Risk," Econometrica, 64(5), 1109-1123.

[36] Gollier, Christian (2001): "Wealth Inequality and Asset Pricing," Review of Economic Studies, 68, 181-203.

[37] Gollier, Christian and Richard J. Zeckhauser (2002): "Horizon Length and Portfolio Risk," Journal of Risk and Uncertainty, 24(3), 195-212.

[38] Gollier, Christian (2004): "Optimal Dynamic Portfolio Risk with First-Order and Second-Order Predictability," The B.E. Journal of Theoretical Economics, 0(1).

[39] Grossman, Sanford J. and Zhongquan Zhou, "Equilibrium Analysis of Portfolio Insurance," Journal of Finance, 51(4),1379-1403.

[40] Hara, Chiaki, Huang, James, and Christoph Kuzmics (2007): "Representative Consumer's Risk Aversion and Efficient Risk-Sharing Rules," Journal of Economic Theory, 137, 652-672.

[41] Harvey, Campbell R. (2001): "The Specification of Conditional Expectations," Journal of Empirical Finance, 8(5), 573-637.

[42] He, Hua, and Hayne Leland (1993): "On Equilibrium Asset Price Processes," Review of Financial Studies, 6(3), 593-617.

[43] He, Hua and Chi-Fu Huang (1994): "Consumption-Portfolio Policies: An Inverse Optimal Problem," Journal of Economic Theory, 62(2), 257-293,

[44] Herbst, Ira and Lauren Pitt (1991): "Diffusion Equation Techniques in Stochastic Monotonicity and Positive Correlations," Probability Theory and Related Fields, 87, 275-312. 
[45] Hugonnier, Julien, Semyon Malamud and Eugene Trubowitz (2009): "Endogenous Completeness of Continuous Time Diffusion Driven Markets," SFI working paper.

[46] Jouini, Elyes and Clotilde Napp (2008): "Consensus Consumer and Intertemporal Asset Pricing with Heterogeneous Beliefs," forthcoming in the Review of Financial Studies.

[47] Kimball, Miles S. (1990): "Precautionary Saving in the Small and in the Large," Econometrica, 58, 53-73.

[48] Kim, Tong Suk and Edward Omberg (1996): "Dynamic Nonmyopic Portfolio Behavior," The Review of Financial Studies, 9(1), 141-161

[49] Kim, Hagen, Hyoung Il Lee, Joon Y. Park and Hyosung Yeo (2009): "Macroeconomic Uncertainty and Asset Prices: A Stochastic Volatility Model," working paper, Texas A\&MM University.

[50] Kogan, Leonid, Stephen Ross, Jiang Wang and Mark Westerfield, (2006): "The Price Impact and Survival of Irrational Traders," Journal of Finance, 61(1), 195-229.

[51] LeRoy, Stephen F. and Robert D. Porter (1981): "The Present-Value Relation: Tests Based on Implied Variance Bounds, Econometrica, 49, 555-574.

[52] Malamud, Semyon (2008): "Long Run Forward Rates and Long Yields of Bonds and Options in Heterogeneous Equilibria," Finance and Stochastics, 12, 245-264.

[53] Mankiw, Greory N., David Romer and Matthew D. Shapiro (1985): "An Unbiased Reexamination of Stock Market Volatility, Journal of Finance, 40, 677-687.

[54] Mankiw, Gregory N. and Matthew D. Shapiro (1986): "Risk and Return: Consumption Beta versus Market Beta," The Review of Economics and Statistics, 68(3), 452-59.

[55] Mankiw, Gregory N., David Romer and Matthew D. Shapiro (1991): "Stock Market Forecastability and Volatility: A Statistical Appraisal," Review of Economic Studies, 58, 455-477.

[56] Mele, Antonio (2007): "Asymmetric Stock Market Volatility and the Cyclical Behavior of Expected Returns," Journal of Financial Economics, 86, 446-478. 
[57] Merton, Robert (1969): "Lifetime Portfolio Selection Under Uncertainty: The Continuous Time Case," Review of Economics and Statistics, 51, 247-257.

[58] Merton, Robert (1971): "Optimum Consumption and Portfolio Rules in a Continuous-time Model," Journal of Economic Theory, 3(4), 373-413.

[59] Merton, Robert (1973): "An Intertemporal Asset Pricing Model," Econometrica, 41, 867-887.

[60] Meulbroek, Lisa (1992): "A Comparison of Forward and Futures Prices of an Interest Rate-Sensitive Financial Asset," Journal of Finance, 47(1), 381-396.

[61] Pratt, John W. (1964): "Risk Aversion in The Small and in the Large," Econometrica, 32, 122-136.

[62] Pratt, John W. and Richard J. Zeckhauser (1987): "Proper Risk Aversion," Econometrica, 55, 143-154.

[63] Rosenberg, Joshua V. and Robert F. Engle (2002): "Empirical Pricing Kernels," Journal of Financial Economics, 64(3), 341-372.

[64] Ross, Stephen A. (1981): "Some Stronger Measures of Risk Aversion in the Small and in the Large with Applications," Econometrica, 49(3), 621-639.

[65] Shiller, Robert J. (1981): "Do Stock Prices Move Too Much to be Justified by Subsequent Changes in Dividends?," American Economic Review, 71, 421-436.

[66] Wachter, Jessica (2002): "Portfolio and Consumption Decisions under MeanReverting Returns: An Exact Solution for Complete Markets," Journal of Financial and Quantitative Analysis, 37, 63-91.

[67] Wang, Jiang (1996): "The Term Structure of Interest Rates in a Pure Exchange Economy with Heterogeneous Investors," Journal of Financial Economics, 41, 75-110.

[68] Wang, Susheng (1993): "The Integrability Problem of Asset Prices," Journal of Economic Theory, 59(1), 199-213.

[69] West, Kenneth D. (1988): "Dividend Innovations and Stock Price Volatility," Econometrica, 56, 37-61. 
[70] Wilson, Robert (1968): "The Theory of Syndicates," Econometrica, 36 (1), 119132. 